\title{
Analysis of the Impact of Different Forecasting Techniques on the Inventory Bullwhip Effect in Two Parallel Supply Chains with a Competition Effect
}

\author{
Xigang Yuan $\mathbb{D}^{1,2}$ Xiaoqing Zhang ${ }^{1}{ }^{1},{ }^{1}$ and Dalin Zhang $^{3}$ \\ ${ }^{1}$ Business School, Jiangsu Normal University, Xuzhou 221116, China \\ ${ }^{2}$ School of Economics and Management School, Southwest Jiaotong University, Chengdu 610031, China \\ ${ }^{3}$ School of Computing Science and Engineering, University of New South Wales, Sydney 2052, Australia \\ Correspondence should be addressed to Xiaoqing Zhang; xqzhang22@163.com
}

Received 31 January 2020; Revised 19 May 2020; Accepted 26 May 2020; Published 11 September 2020

Academic Editor: Chuan-Yu Wu

Copyright (c) 2020 Xigang Yuan et al. This is an open access article distributed under the Creative Commons Attribution License, which permits unrestricted use, distribution, and reproduction in any medium, provided the original work is properly cited.

This paper studies the impact of different forecasting techniques on the inventory bullwhip effect in two parallel supply chains with the competition effect, which is in contrast to the situation of a single product in a serial supply chain. In particular, this paper constructs two parallel supply chains, each of which includes one manufacturer and one retailer. Moreover, the market demand is impacted by the self-price sensitivity coefficient, the cross-price sensitivity coefficient, the market share, and the demand shock. We then assumed that the retailer can forecast market demand by using different forecasting techniques (i.e., the moving average technique (MA), the exponential smoothing technique (ES), and the minimum mean square error technique (MMSE)). We constructed the quantity model of the bullwhip effect and the inventory bullwhip effect. Finally, we analyzed the impact of different forecasting techniques and market share on the inventory bullwhip effect. We analyzed the conditions under which the retailers should choose different types of forecasting techniques on the basis of the inventory bullwhip effect. The results show that the MMSE forecasting technique can reduce the lead-time demand forecast error to the largest extent, and the inventory bullwhip effect can obtain the lowest level using the MMSE method: retailer-1 can reduce the inventory bullwhip effect by using the MA technique, when the self-price sensitivity coefficient, the price autoregressive coefficient, and the probabilities associated with customers choosing retailer-1's product are very low.

\section{Introduction}

Demand amplification is the primary obstacle to achieving coordination and maintaining harmony at different stages of supply chains and is termed as the bullwhip effect (BWE). The bullwhip is the phenomenon of information distortion as ordering information percolates upstream, which means that a downstream demand fluctuation will lead to a larger fluctuation in the variance of upstream ordering $[1,2]$. The application of the simple two-level supply chain modeling assumption is a widespread method for studying the bullwhip effect (e.g., [1, 3-6]). However, with the development of the economy and society, in many industries (e.g., car manufacturing, electronic enterprises, and mobile phone manufacturers), the traditional model of a simple two-level supply chain, which is used to address the issue of the bullwhip effect, is evolving into a new model that reflects a chain-to-chain competition effect which is caused by the substitutability of homogeneous products. This competition effect means that the firm is not only affected by others within the same supply chain, but is also affected by firms from other supply chains.

In this paper, the inventory bullwhip effect is used as a measure of supply chain performance, and we examined the industry with respect to two parallel supply chains that show a competition effect at the level of their demand stream. Large numbers of industries satisfy the standard of our model. For example, in the mobile phone industry, which includes Huawei and Oppo, each company can form a chain relationship with its downstream retailer, who shares the 
same demand market. It is well-known that the selling price of mobile phones is an important factor that affects the customer demand. For example, the higher the selling price of a Huawei mobile phone, the lower the market demand will be, and vice versa. However, due to the substitutability between Huawei and Oppo, if the Oppo mobile phone's selling price is too high, many customers will turn to Huawei, and Huawei's market share will increase. On the other hand, if Huawei mobile phone's selling price is too high, many customers will turn to Oppo, and Oppo's market share will increase. In other words, customers make purchasing decisions based not only on Oppo's selling price but also on Huawei's selling price.

The competition effect will impact the performance of a simple two-level supply chain. However, it is difficult to determine the competition effect, particularly in terms of whether or not firms will benefit from the inventory bullwhip effect. Only a limited number of papers discuss the problem of the inventory bullwhip effect from the perspective of the two-level supply chain. For example, Ma et al. [7] derived the analytical expressions of the bullwhip effect on product orders and inventory using the minimum mean squared error (MMSE), moving average (MA), and exponential smoothing (ES) forecasting techniques in a simple two-level supply chain with one manufacturer and one retailer. However, they did not discuss the impact of different forecasting techniques on the inventory bullwhip effect in two parallel supply chain systems. On the contrary, we extend the work of Ma et al. [7], who considered a twolevel supply chain in which the demand was price sensitive, by quantifying the bullwhip effect on inventory (i.e., the increase in inventory variability). We elaborate upon that work by considering two parallel supply chains, in which each sells a single product and each includes one manufacturer and one retailer, such that the competition effect exists in the two parallel supply chains, and the demand that both retailers experience is price sensitive. The price follows dynamics with an autoregressive $\mathrm{AR}(1)$ pricing process, and the competition effect and the market share follow the demand process. Based on the analysis, we derive the mathematical modeling of the inventory bullwhip effect by using MA, ES, and MMSE forecasting techniques. Moreover, we investigated the impact of the competition effect and the market share on the inventory bullwhip effect under different forecasting techniques. Finally, we explored the condition under which the retailer chooses the best forecasting technique to minimize the inventory bullwhip effect.

The remainder of this paper is organized as follows: Section 2 reviews the relevant literature. Section 3 presents the parameters and modeling assumption and the problem. Section 4 deduces the bullwhip effect (BWE) and the inventory bullwhip effect (IBWE). Section 5 discusses the impact of different forecasting techniques on the IBWE and explores the condition under which the retailer chooses the best forecasting technique to minimize the IBWE. The final section presents the conclusion which outlines the limitations of this paper and the directions for future research.

\section{Literature Review}

In this section, we review the existing literature from the following three perspectives: (1) bullwhip effect in a twolevel supply chain; (2) bullwhip effect in a multilevel supply chain; (3) bullwhip effect in a two-level supply chain network.

2.1. Bullwhip Effect in a Two-Level Supply Chain. The bullwhip effect is a widespread phenomenon in the supply chain, which means that a downstream demand fluctuation will lead to a larger fluctuation in the variance of upstream ordering $[1,2]$. This phenomenon can lead to a vast amount of problems, such as superfluous inventory, erroneous product forecasts, and insufficient or excessive capacities [2]. Therefore, in recent years, it has attracted the attention of numerous administrators and researchers. Many studies quantify the bullwhip effect and determine measures by which it can be reduced in a two-level supply chain. Lee et al. [1], and Lee et al. [8] discussed the bullwhip effect in a twolevel supply chain which consisted of one manufacturer and one retailer and followed the dynamics of a first-order autoregressive demand $(\operatorname{AR}(1))$ process by using the moving average forecasting technique. The result has shown that the moving average forecasting technique could reduce the bullwhip effect. Alwan et al. [9], Hausman and Miyaoka [10], and Liu and Wang [11] researched the bullwhip effect in a two-level supply chain under the $\operatorname{ARMA}(1,1)$ demand process by using the exponential smoothing method. They compared the different influence between these two kinds of forecasting techniques. In addition, the $\operatorname{ARMA}(p, q)$ demand process was discussed by Gaalman and Disney [12] and Gaalman et al. [13] with the moving average forecasting technique. These aforementioned studies discussed how the bullwhip effect can be quantified by using different autoregressive demand processes in a two-level supply chain which consisted of one supplier and one retailer or one manufacturer and one retailer. Moreover, they discussed the impact of different forecasting techniques on the bullwhip effect in the two-level supply chain.

In addition, many scholars discussed the bullwhip effect in a two-level supply chain using the control approach. Holt et al. [14] developed the HMMS control model in a two-level supply chain and pointed out that this model can effectively balance the relationship between ordering from the retailer and ordering from the supplier. Blinder et al. [15] proposed the reduction of the bullwhip effect by using the $(\mathrm{S}, \mathrm{S})$ ordering strategy. Baganha and Cohen [16] designed a particular inventory control strategy and pointed out that it could reduce the fluctuation of demand information. In addition, other scholars studied the bullwhip effect using the discrete control theory, $H_{\propto}$ control theory, control-based forecasting technique, and O-S feedback control method in a two-level supply chain (e.g., [17-22]). Udenio et al. [23] analyzed the bullwhip effect using the system dynamics (SD) model. In addition, many scholars study the bullwhip effect in the two-level supply chain using different kinds of forecasting techniques. Chen et al. [4] proposed the causal of 
the bullwhip effect using the exponential smoothing method and compared the result with the increase in variability due to the use of the moving average forecasting technique. Kim and Ryan [24] analyzed the impact of bullwhip on inventory using the exponential smoothing forecasting technique. Michna et al. [25] quantified the bullwhip effect (which measures how the variance of replenishment orders is amplified as the orders move up the supply chain) when both random demands and random lead times were estimated using the moving average forecasting method in the twolevel supply chain. They found that maxima and minima in the bullwhip measure as a function of the demand autocorrelation. Erica et al. [26] studied a two-echelon singleproduct supply chain with final demand distributed according to a known $\mathrm{AR}(1)$ process but with unknown parameters. The results have shown that the bullwhip effect was affected by unknown parameters and was influenced by the frequency with which parameter estimates were updated.

\subsection{Bullwhip Effect in a Multilevel Supply Chain. With} economic and societal development, more and more enterprises are involved in the supply chain, and the two-level supply chain structure evolves into a multilevel supply chain structure. Many scholars attempted to analyze the bullwhip effect in a multilevel supply chain using different forecasting techniques. Holland et al. [27] pointed out that price fluctuations could cause the bullwhip effect by using the exponential smoothing forecasting technique. Zhang [28, 29] analyzed the bullwhip effect using the minimize mean square error forecasting technique and compared the results with the moving average technique and exponential smoothing techniques, respectively. Hosoda and Disney [30] studied the impact of the bullwhip effect on inventory using the minimize mean square error forecasting technique and found that it could reduce the impact of bullwhip effect on inventory. Lu et al. [31] simulated the forming process of the bullwhip in a multilevel supply chain. Cachon et al. [32] collected the microeconomic industry-level US data and observed that manufacturing industries did not have substantially greater demand volatility than retail industries. Liu and Wang [33] formulated the mathematical expression of the bullwhip effect in a multilevel supply chain using the mean square error forecasting technique and compared with the moving average forecasting technique.

Liu and Wang [33] analyzed the bullwhip of a multilevel supply chain by using the exponential smoothing forecasting technique and the minimize mean square error method, respectively. Kim and Springer [34] studied demand volatility by employing a system dynamic model. In a follow-up study, Springer and Kim [35] also analyzed the inventory volatility using a system dynamic model in a multilevel supply chain. They pointed out that the inventory volatility was caused by the demand volatility. Coppini et al. [36] simulated the four-stage beer game supply chain model, and they showed that inventory oscillations provided more information on supply chain performance than the bullwhip effect on product orders. Some other forecasting techniques have also been adopted by Ma et al. [37]. Costantino et al.
[38] studied the impact of demand sharing on the order strategy using the simulation method. They found that the demand sharing helped to reduce the order volatility. Robert and Haim [39] estimated both a strong bullwhip and robust smoothing and found that firms smooth both production variability and production uncertainty. Hossein et al. [40] quantified the bullwhip effect, order rate variance ratio (OVR), and inventory variance ratio (IV) in a three-stage supply chain with multiple retailers. Moreover, they surveyed the influence of the correlation coefficient on the bullwhip effect. Marieh and Mohsen [41] investigated the measure of the bullwhip effect in three different supply chains. Moreover, they pointed out that these three different supply chains were subsequently analyzed to determine which supply chain helps to reduce the bullwhip effect more. Zhu et al. [42] investigated the factors that impacted the bullwhip effect in the oil and gas supply chain using case study evidence from six companies in North America, which cover refining and marketing, exploration and production, integrated oil and gas, and drilling. The findings indicated that the existing theories of the bullwhip effect had limitations in explaining the phenomenon in the oil and gas industry.

\subsection{Bullwhip Effect in a Two-Level Supply Chain Network.} The simple two-level supply chain modeling assumption has been widely used to study the bullwhip effect. On the contrary, due to the complexity of the supply chain network's structure, the simple two-level supply chain modeling assumption is outdated. Recently, some scholars discussed the bullwhip effect in a two-level supply chain network [43-45]. However, most of these studies assumed that customer demand follows the AR(1) autoregressive process, and they mainly discuss supply chain coordination and how to reduce the bullwhip effect. Zhang and Yuan [44] proposed that the-old-for-new policy could reduce the bullwhip effect and could increase profitability in a closed-loop supply chain network when the firm could forecast the customer's demand by using the moving average forecasting technique. Yuan and Zhu [45] provided three quantitative models of the bullwhip effect in a two-level supply chain network consisting of a single manufacturer and two retailers. Moreover, they compared the different impact of forecasting techniques on the bullwhip effect. Zhang and Zhang [46] addressed a fuzzy robust control (FRC) approach to mitigate the bullwhip effect in the uncertain closed-loop supply chain with lead times. A new FRC approach was proposed to mitigate the bullwhip effect and realize the robust stability of the uncertain closed-loop supply chain with lead times. A simulation example verified the mitigation effect of the bullwhip effect under the proposed FRC approach.

Following the work by Yuan and Zhu [45], we extended the research of $\mathrm{Ma}$ et al. [7] and developed a theoretical framework involving two parallel supply chains in which the competition effect exists due to product substitutability, and the two supply chains consist of one manufacturer and one retailer. We discuss the impact of different forecasting techniques on the inventory bullwhip effect and explore the 
condition under which the inventory bullwhip effect is amplified (or reduced) in relation to a situation without competition and market share.

Though many scholars study the bullwhip effect, extensive problems still need to be resolved, such as the issue related to how the bullwhip effect can be quantified and reduced in a complex supply chain network. From the above analysis, the previous studies have many limitations: (1) the objective is mainly concentrated on the two-level or multilevel supply chain, and few scholars considered twolevel or multilevel supply chain networks that consist of two manufacturers and two retailers; (2) many scholars assumed that they trade one kind of product between different firms, and fewer studies considered trades in two different kinds of products which are substitutable between different firms; (3) most studies discussed the impact of lead time, the moving average period, and other factors on the bullwhip effect, and fewer researchers studied the impact of different kinds of forecasting techniques on the inventory bullwhip effect.

The major contribution of this paper is as follows: (1) the research object of this paper mainly involves two parallel supply chains consisting of two manufacturers and two retailers; (2) we assumed that demand is price sensitive, and that the price follows a first-order autoregressive pricing process. We assumed that two retailers (R1 and R2) share the market, and thus, we introduced the self-price sensitivity coefficient, the cross-price sensitivity coefficient, and the market share into the demand model. Moreover, we developed a quantitative model of the inventory bullwhip effect in two parallel supply chains; (3) we analyzed the impact of different forecasting techniques on the inventory bullwhip effect.

\section{Parameters and Modeling Assumption and Problem Description}

3.1. Description of Parameters. In this section, we describe and explain the relevant variables, as shown in Table 1.

3.2. Modeling Assumption. In this section, in order to make the mathematical expression of the bullwhip effect and the inventory bullwhip effect more meaningful in a practical way, we made the following assumption:

(1) There are only two supply chains in the market, each of which consists of one manufacturer and one retailer, represented as $M_{i}$ and $R_{i}(i=1,2)$, respectively, and they only trade one kind of product in each of the supply chains;

(2) Two retailers (R1 and R2) sell two different kinds of products which are substitutable;

The probabilities that customers would choose R1's product and R2's product are expressed as $\psi$ and $1-\psi$, respectively;

(3) The demand that is experienced by $\mathrm{R} 1$ is impacted by R1's selling price and R2's selling price. The demand
TABLE 1: Relevant parameters and explanations.

\begin{tabular}{|c|c|}
\hline Parameters & Explanations \\
\hline$a_{1}$ & Retailer-one (R1) potential market demand \\
\hline$a_{2}$ & Retailer-two (R2) potential market demand \\
\hline$b_{11}^{2}$ & R1 self-price sensitivity coefficient \\
\hline$b_{12}$ & $\mathrm{R} 1$ cross-price sensitivity coefficient \\
\hline$b_{21}$ & R2 self-price sensitivity coefficient \\
\hline$b_{22}$ & $\mathrm{R} 2$ cross-price sensitivity coefficient \\
\hline$\psi$ & $\begin{array}{c}\text { The probabilities that customers will choose R1's } \\
\text { product }\end{array}$ \\
\hline $1-\psi$ & $\begin{array}{l}\text { The probabilities for customers choosing R2's } \\
\text { product }\end{array}$ \\
\hline$\rho_{1}$ & R1 price autoregressive coefficient \\
\hline$\rho_{2}$ & $\mathrm{R} 2$ price autoregressive coefficient \\
\hline$\mu_{1, p}$ & The expectation of $p_{t}^{1}$ \\
\hline$\mu_{2, p}$ & The expectation of $p_{t}^{2}$ \\
\hline$\delta_{1, p}^{2}$ & The variance of $p_{t}^{1}$ \\
\hline$\delta_{2, p}^{2}$ & The variance of $p_{t}^{2}$ \\
\hline$\mu_{1, d}$ & The expectation of $d_{1, t}$ \\
\hline$\mu_{2, d}$ & The expectation of $d_{2, t}$ \\
\hline$\delta_{1, d}^{2}$ & The variance of $d_{1, t}$ \\
\hline$\delta_{2, d}^{2}$ & The variance of $d_{2, t}$ \\
\hline$q_{1, t}^{2, u}$ & R1 order quantity \\
\hline$q_{2, t}$ & $\mathrm{R} 2$ order quantity \\
\hline$l_{1}$ & $\mathrm{R} 1$ lead time \\
\hline$l_{2}$ & R2 lead time \\
\hline$\widehat{\xi}_{1, t}^{l_{1}}$ & $\begin{array}{l}\text { The estimate of the standard deviation of the } l_{1} \\
\text { period forecast error of R1 }\end{array}$ \\
\hline$\widehat{\xi}_{2, t}^{2}$ & $\begin{array}{l}\text { The estimate of the standard deviation of the } l_{2} \\
\text { period forecast error of R2 }\end{array}$ \\
\hline$\widehat{d}_{1, t}^{l_{1}}$ & R1 mean lead-time demand \\
\hline$\widehat{d}_{2, t}^{l_{2}^{*}}$ & R2 mean lead-time demand \\
\hline$I_{1, t}^{2, t}$ & R1 inventory level \\
\hline$I_{2, t}$ & R2 inventory level \\
\hline$\alpha$ & Smoothing constant \\
\hline$k$ & The moving average period \\
\hline
\end{tabular}

that is faced by $\mathrm{R} 2$ is impacted by R2's selling price and R1's selling price [7, 37];

(4) We assumed that demand is price sensitive and that the price follows the dynamics of the AR(1) autoregressive pricing process, and in the price models, we considered the probability that customers would choose both retailers' products [47];

(5) We only developed the mathematical expression of the bullwhip effect and the inventory bullwhip effect for two retailers. Thus, we assumed that retailers and manufacturers do not share end-demand information. The retailer forecasts customer demand using customer demand information, while the manufacturer forecasts retailer demand using the retailer's order information;

(6) The lead time $l_{1}$ and $l_{2}$ are fixed, and the order is received at the start of period $t+l_{1}+1$ and $t+l_{2}+1$, respectively. Both retailers meet the customer demand $d_{1, t}$ and $d_{2, t}$ and backlog any excessive demand, respectively. At the same time, it was assumed that the demand of both retailers are two independent variables. 
3.3. Problem Description. In this section, there are only two supply chains in the market, each of which consists of one manufacturer and one retailer, denoted by $M_{i}$ and $R_{i}$ $(i=1,2)$, respectively. Two retailers are responsible for meeting the needs of customers. Two manufacturers are responsible for supplying the product to two retailers. The demand information flows downstream to upstream, and the inventory information flows upstream to downstream. The theoretical model of the two parallel supply chains can be seen in Figure 1. Two retailers share the same market, and the differences between the two retailers' market prices can have an impact on the customer's purchasing behavior. We assumed that the probability of customers choosing both R1's product and R2's product can be expressed as $\psi$ and $1-\psi$, respectively. At the end of period $t$, two retailers place an order, i.e., $q_{1, t}$ and $q_{2, t}$, to two manufacturers, respectively.

Two retailers face the same demand process, such that the demand that is experienced by the two retailers' is impacted by R1's selling price and R2's selling price. The following basic linear demand function model of the two retailers can be obtained:

$$
\begin{aligned}
& d_{1, t}=a_{1}-b_{11} p_{1, t}+b_{12} p_{2, t}+\varepsilon_{1, t}, \\
& d_{2, t}=a_{2}-b_{21} p_{2, t}+b_{22} p_{1, t}+\varepsilon_{2, t},
\end{aligned}
$$

where $a_{1}$ and $a_{2}$ represent the potential market demand which are nonnegative constants, $b_{11}$ and $b_{21}$ denote the self-price sensitivity coefficients which are nonnegative constants, $b_{12}$ and $b_{22}$ are the cross-price sensitivity coefficients, and $\varepsilon_{1, t}$ and $\varepsilon_{2, t}$ are the independent and identically normally distributed demand shocks with a zero mean and variance of $\sigma_{1}^{2}$ and $\sigma_{2}^{2}$. It is important to emphasize that $b_{12}$ and $b_{22}$ are nonnegative because the two types of products are substitutable. From equation (1), we can see that the potential market demand, the self-price sensitivity coefficient, the cross-price sensitivity coefficients, and the demand shock are considered in the demand model. Thus, this makes the demand function more significant in practical terms.

We assumed that the retailer trades on a perfectly competitive market and exerts no control over the market clearing price. Let market price $p_{1, t}$ and $p_{2, t}$ in equation (1) be an $\mathrm{AR}(1)$ pricing process. The probability that customers will choose both retailers' products is added to the AR(1) pricing process (the same pricing process can be seen in [7]):

$$
\begin{aligned}
& p_{1, t}=\psi \mu_{1}+\rho_{1} p_{1, t-1}+\psi \eta_{1, t}, \\
& p_{2, t}=(1-\psi) \mu_{2}+\rho_{2} p_{2, t-1}+(1-\psi) \eta_{2, t},
\end{aligned}
$$

where $\mu_{1}$ and $\mu_{2}$ are the nonnegative constants, $\rho_{1}$ and $\rho_{2}$ are the price autoregressive coefficients which satisfy $0<\rho_{1}<1$ and $0<\rho_{2}<1$, and $\eta_{1, t}$ and $\eta_{2, t}$ are the independent and identically normally distributed price shocks with a zero mean and variance of $\delta_{1}^{2}$ and $\delta_{2}^{2}$. From equations (1) and (2), we can obtain $d_{1, t}=a_{1}^{\prime}-b_{11}^{\prime} p_{1, t-1}+b_{12}^{\prime} p_{2, t-1}+\varepsilon_{1, t-1}^{\prime}$, where $a_{1}^{\prime}=a_{1}, b_{11}^{\prime}=b_{11} \rho_{1}$, and $b_{12}^{\prime}=b_{12} \rho_{2}$.

$\varepsilon_{1, t-1}=\varepsilon_{1, t-1}^{\prime}-b_{11} \psi u_{1}-b_{11} \psi \eta_{1, t}+b_{12}(1-\psi) u_{2}+b_{12}(1-$ భ) $\eta_{2, t}$. We can see that the demand model is not an AR(1) process. On the contrary, the $\mathrm{AR}(1)$ demand process is used

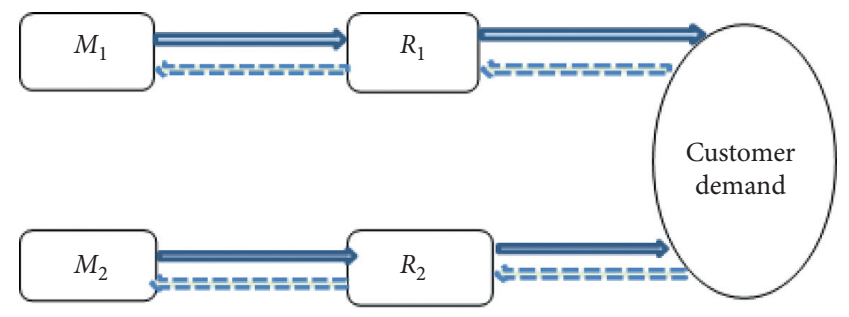

Inventory information flow Demand information flow

FIgURE 1: The theoretical model of the two parallel supply chains.

by most scholars (e.g., $[1,3])$, and it is difficult to explain its managerial insights. Thus, we assumed that the price-sensitive demand function and the price are an $\mathrm{AR}(1)$ process. Thus, the self-price sensitivity coefficient, the cross-price sensitivity coefficients, and the demand shock are inputted into the demand model. The covariance of the price shock can be expressed as

$$
\operatorname{Cov}\left(\eta_{1, t}, \eta_{2, t}\right)= \begin{cases}\delta_{12}^{2}, & t=t^{\prime}, \\ 0, & t \neq t^{\prime}\end{cases}
$$

Based on the property of the $\mathrm{AR}(1)$ process, in any period $t$, the expectation and the variance of $p_{t}^{1}$ and $p_{t}^{2}$ can be expressed as $\mu_{1, p}=E\left(p_{1, t}\right)=\psi \mu_{1} / 1-\rho_{1} ; \mu_{2, p}=E\left(p_{2, t}\right)=$ $(1-\psi) \mu_{2} / 1-\rho_{2}$ and as $\delta_{1, p}^{2}=\operatorname{Var}\left(p_{1, t}\right)=\psi^{2} \mu_{1}^{2} / 1-\rho_{1}^{2}$; $\delta_{2, p}^{2}=\operatorname{Var}\left(p_{2, t}\right)=(1-\psi)^{2} \mu_{2}^{2} / 1-\rho_{2}^{2}$. Moreover, the expectation and the variance of $d_{1, t}$ and $d_{2, t}$ can be expressed as $\mu_{1, d}=E\left(d_{1, t}\right)=a_{1}-b_{11} \mu_{1, p}+b_{12} \mu_{2, p} ; \mu_{2, d}=E\left(d_{2, t}\right)=a_{2}-$ $b_{21} \mu_{2, p}+b_{22} \mu_{1, p} ; \quad \delta_{1, d}^{2}=\operatorname{Var}\left(d_{1, t}\right)=\sigma_{1}^{2}+b_{11}^{2} \delta_{1, p}^{2}+b_{12}^{2} \delta_{2, p}^{2}-$ $2 b_{11} b_{12} \psi(1-\psi) \delta_{12}^{2} / 1-\rho_{1} \rho_{2} ; \delta_{2, d}^{2}=\operatorname{Var}\left(d_{2, t}\right)=\sigma_{2}^{2}+b_{21}^{2} \delta_{2, p}^{2}$ $+b_{22}^{2} \delta_{1, p}^{2}-2 b_{21} b_{22} \psi(1-\psi) \delta_{12}^{2} / 1-\rho_{1} \rho_{2}$.

\section{Ordering Process of the Two Retailers}

In this section, we assume that the two retailers have utilized the most common inventory strategy, i.e., the order-up-to strategy. R1 and R2 can determine customer demand $d_{1, t-1}$ and $d_{2, t-1}$ at the end of period $t-1$. The two retailers then place an order of quantity $q_{1, t}$ and $q_{2, t}$ to two manufacturers at the beginning of period $t$. We proposed that R1 and R2's lead time $l_{1}$ and $l_{2}$ is fixed. Both retailers can receive the product at the beginning of the period $t+l_{1}$ and $t+l_{2}$, respectively. Thus, $q_{1, t}$ and $q_{2, t}$ can be calculated relative to the demand $d_{1, t}$ and $d_{2, t}$ as follows:

$$
\begin{aligned}
& q_{1, t}=S_{1, t}-S_{1, t-1}+d_{1, t-1}, \\
& q_{2, t}=S_{2, t}-S_{2, t-1}+d_{2, t-1},
\end{aligned}
$$

where $S_{1, t}$ and $S_{2, t}$ represent the highest inventory levels for the two retailers, and these levels are set to satisfy the goal of the inventory strategy. They are estimated from the observed demand as follows:

$$
\begin{aligned}
& S_{1, t}=\hat{d}_{1, t}^{l_{1}}+z_{1} \hat{\xi}_{1, t}^{l_{1}}, \\
& S_{2, t}=\hat{d}_{2, t}^{l_{2}}+z_{2} \hat{\xi}_{2 t}^{l_{2}},
\end{aligned}
$$


where $\hat{d}_{1, t}^{l_{1}}$ and $\hat{d}_{2, t}^{l_{2}}$ are the two estimates of the mean leadtime demands using different forecasting techniques (i.e., moving average technique (MA), exponential smoothing technique (ES), and minimum mean square error technique (MMSE)), $z_{1}$ and $z_{2}$ are the two constants to satisfy a desired service level, and $\hat{\xi}_{1, t}^{l_{1}}$ and $\hat{\xi}_{2, t}^{l_{2}}$ are the two estimates of the standard deviation of the $l_{1}$ and $l_{2}$ period forecast errors of $\mathrm{R} 1$ and R2, respectively. Thus, the order quantity $q_{1, t}$ and $q_{2, t}$ for both retailers can be calculated relative to the estimates of the lead-time demands $\widehat{d}_{1, t}^{l_{1}}$ and $\widehat{d}_{2, t}^{l_{2}}$ as

$$
\begin{aligned}
& q_{1, t}=\widehat{d}_{1, t}^{l_{1}}-\widehat{d}_{1, t-1}^{l_{1}}+d_{1, t-1}+z_{1}\left(\widehat{\xi}_{1, t}^{l_{1}}-\hat{\xi}_{1, t-1}^{l_{1}}\right), \\
& q_{2, t}=\widehat{d}_{2, t}^{l_{2}}-\widehat{d}_{2, t-1}^{l_{2}}+d_{2, t-1}+z_{2}\left(\hat{\xi}_{2, t}^{l_{2}}-\hat{\xi}_{2, t-1}^{l_{2}}\right) .
\end{aligned}
$$

\section{The Inventory Bullwhip Effect under Different Forecasting Techniques}

Ma et al. [7] research discussed the impact of the bullwhip effect on product orders and inventory in two-level supply chains which included one manufacturer and one retailer. In contrast, in this section, we mainly discuss the influence of different forecasting techniques on the inventory bullwhip effect in two parallel supply chains which include two manufacturers and two retailers. First, we developed a quantitative model of the inventory bullwhip effect (IBE) under different forecasting techniques. We analyzed the conditions under which the retailers should choose different forecasting techniques on the basis of the inventory bullwhip effect.

5.1. The Inventory Bullwhip Effect Using the MA Techniques. In equation (6), we can determine the mean lead-time demands, i.e., $\hat{d}_{1, t}^{l_{1}}$ and $\hat{d}_{2, t}^{l_{2}}$, using the MA technique, respectively, where $k$ is the moving average period:

$$
\begin{aligned}
& \hat{d}_{1, t}^{l_{1}}=l_{1}\left(\frac{\sum_{i=1}^{p_{1}} d_{1, t-i}}{k}\right), \\
& \hat{d}_{2, t}^{l_{2}}=l_{2}\left(\frac{\sum_{i=1}^{p_{2}} d_{2, t-i}}{k}\right) .
\end{aligned}
$$

Then, $q_{1, t}$ and $q_{2, t}$ in equation (6) can be formulated as equation (8):

$$
\begin{aligned}
& q_{1, t}=\left(1+\frac{l_{1}}{k}\right) d_{1, t-1}-\left(\frac{l_{1}}{k}\right) d_{1, t-k-1}+z_{1}\left(\hat{\xi}_{1, t}^{l_{1}}-\hat{\xi}_{1, t-1}^{l_{1}}\right), \\
& q_{2, t}==\left(1+\frac{l_{2}}{k}\right) d_{2, t-1}-\left(\frac{l_{2}}{k}\right) d_{2, t-k-1}+z_{2}\left(\hat{\xi}_{2, t}^{l_{2}}-\widehat{\xi}_{2, t-1}^{l_{2}}\right) .
\end{aligned}
$$

Thus, the variance of the order quantity for R1 and R2 can be derived as follows:

$$
\begin{aligned}
\operatorname{Var}\left(q_{1, t}\right)= & {\left[\left(1+\Lambda_{1}\right)^{2}+\Lambda_{1}^{2}\right]\left[\sigma_{1}^{2}+b_{11}^{2} \operatorname{Var}\left(p_{1, t}\right)+b_{12}^{2} \operatorname{Var}\left(p_{2, t}\right)-2 b_{11} b_{12} \delta_{12}^{2} \Pi\right] } \\
& -2\left(1+\Lambda_{1}\right)\left(\Lambda_{1}\right)\left\{b_{11}^{2} \operatorname{Var}\left(p_{1, t}\right)+b_{12}^{2} \operatorname{Var}\left(p_{2, t}\right)-b_{11} b_{12}\left(\rho_{1}^{k}+\rho_{2}^{k}\right) \delta_{12}^{2} \Pi\right\} \\
\operatorname{Var}\left(q_{2, t}\right)= & {\left[\left(1+\Lambda_{2}\right)^{2}+\Lambda_{2}^{2}\right]\left[\sigma_{2}^{2}+b_{21}^{2} \operatorname{Var}\left(p_{2, t}\right)+b_{22}^{2} \operatorname{Var}\left(p_{1, t}\right)-2 b_{21} b_{22} \delta_{12}^{2} \Pi\right] } \\
& -2\left(1+\Lambda_{2}\right)\left(\Lambda_{2}\right)\left\{b_{21}^{2} \rho_{2}^{k} \operatorname{Var}\left(p_{2, t}\right)+b_{22}^{2} \rho_{1}^{k} \operatorname{Var}\left(p_{1, t}\right)-b_{21} b_{22}\left(\rho_{2}^{k}+\rho_{1}^{k}\right) \delta_{12}^{2} \Pi\right\},
\end{aligned}
$$

where $\Lambda_{1}=l_{1} / k, \Lambda_{2}=l_{2} / k$, and $\Pi=(\psi(1-\psi)) /\left(1-\rho_{1} \rho_{2}\right)$.

Theorem 1. For two parallel supply chains in which there is a competition effect on customer demand and the retailers' market share, when two retailers use the order-up-to inventory strategy and the MA forecasting technique, the quantitative model of the bullwhip effect in two supply chains is expressed as follows:

$$
\begin{aligned}
& \mathrm{BE}_{\text {retailer-1 }}^{\mathrm{MA}}=\frac{\operatorname{Var}\left(q_{1, t}\right)}{\operatorname{Var}\left(d_{1, t}\right)}=1+\frac{\left[2 \Lambda_{1}^{2}+2 \Lambda_{1}\right]\left[\sigma_{1}^{2}+b_{11}^{2} \delta_{1, p}^{2}+b_{12}^{2} \delta_{2, p}^{2}-2 b_{11} b_{12} \delta_{12}^{2} \Delta\right]-2\left(1+\Lambda_{1}\right)\left(\Lambda_{1}\right)\left\{b_{11}^{2} \delta_{1, p}^{2}+b_{12}^{2} \delta_{2, p}^{2}-b_{11} b_{12}\left(\rho_{1}^{k}+\rho_{2}^{k}\right) \delta_{12}^{2} \Delta\right\}}{\delta_{1, d}^{2}}, \\
& \mathrm{BE}_{\text {retailer-2 }}^{\mathrm{MA}}=\frac{\operatorname{Var}\left(q_{2, t}\right)}{\operatorname{Var}\left(d_{2, t}\right)}=1+\frac{\left[2 \Lambda_{2}^{2}+2 \Lambda_{2}\right]\left[\sigma_{2}^{2}+b_{21}^{2} \delta_{2, p}^{2}+b_{22}^{2} \delta_{1, p}^{2}-2 b_{21} b_{22} \delta_{12}^{2} \Delta\right]-2\left(1+\Lambda_{2}\right)\left(\Lambda_{2}\right)\left\{b_{21}^{2} \rho_{2}^{k} \delta_{2, p}^{2}+b_{22}^{2} \rho_{1}^{k} \delta_{1, p}^{2}-b_{21} b_{22}\left(\rho_{2}^{k}+\rho_{1}^{k}\right) \delta_{12}^{2} \Delta\right\}}{\delta_{2, d}^{2}},
\end{aligned}
$$

where $\Lambda_{1}=l_{1} / k, \Lambda_{2}=l_{2} / k$, and $\Delta=(\psi(1-\psi)) /\left(1-\rho_{1} \rho_{2}\right)$.

In order to analyze the impact of the bullwhip effect on the inventory level, we calculated the variance of the inventory level for the two retailers at period $t$ as $I_{1, t}$ and
$I_{2, t}$, corresponding to the bullwhip effect. $I_{1, t}$ and $I_{2, t}$ can be expressed as follows:

$$
\begin{aligned}
& I_{1, t}=I_{1, t-1}+q_{1, t-l_{1}}-d_{1, t-1}, \\
& I_{2, t}=I_{2, t-1}+q_{2, t-l_{2}}-d_{2, t-1} .
\end{aligned}
$$


From equation (11), the following can be obtained:

$$
\begin{aligned}
& q_{1, t-l_{1}}=I_{1, t}-I_{1, t-1}+d_{1, t-1}, \\
& q_{2, t-l_{2}}=I_{2, t}-I_{2, t-1}+d_{2, t-1} .
\end{aligned}
$$

Vassian [48] proposed that $q_{1, t-l_{1}}$ and $q_{2, t-l_{2}}$ can be expressed as follows:

$$
q_{1, t-l_{1}}=\widehat{d}_{1, t}^{l_{1}}-\sum_{i=1}^{l_{1}-1} q_{1, t-i}-I_{1, t}, q_{2, t-l_{2}}=\widehat{d}_{2, t}^{l_{2}}-\sum_{i=1}^{l_{2}-1} q_{2, t-i}-I_{2, t} .
$$

We substituted equation (13) into equation (12), and we can thus obtain equation (14) as follows:

$$
I_{1, t}=\widehat{\xi}_{1, t}^{l_{1}}=\widehat{d}_{1, t-l_{1}}^{l_{1}}-d_{1, t-l_{1}}^{l_{1}}, I_{2, t}=\widehat{\xi}_{2, t}^{l_{2}}=\widehat{d}_{2, t-l_{2}}^{l_{2}}-d_{2, t-l_{2}}^{l_{2}} .
$$

Lemma 1. Using the MA forecasting technique, the estimate of the standard deviation of the $l_{1}$ period forecast error of $R 1 \widehat{\xi}_{1, t}^{l_{1}}$ is a constant and can be expressed as follows:

$$
\hat{\xi}_{1, t}^{l_{1}}=\sqrt{\begin{array}{l}
l_{1} \delta_{1, d}^{2}+2 b_{11}^{2} \delta_{1, p}^{2} \Lambda_{1}\left(l_{1}-\Omega_{1}\right)-2 b_{11} b_{12} \delta_{12}^{2} \Delta \Lambda_{1}\left(l_{1}-\Omega_{1}\right)-2 b_{12} b_{11} \delta_{12}^{2} \Delta \Lambda_{2}\left(l_{2}-\Omega_{2}\right)+2 b_{12}^{2} \delta_{2, p}^{2} \Lambda_{2}\left(l_{2}-\Omega_{2}\right) \\
+\left(\frac{l_{1}}{k}\right)^{2}\left[k \delta_{1, d}^{2}+2 b_{11}^{2} \delta_{1, p}^{2} \Lambda_{1}\left(k-\Theta_{1}\right)+2 b_{12}^{2} \delta_{2, p}^{2} \Lambda_{2}\left(k-\Theta_{2}\right)-2 b_{11} b_{12}\left(\Lambda_{1}\left(k-\Theta_{1}\right)+\Lambda_{2}\left(k-\Theta_{2}\right)\right) \Delta \delta_{12}^{2}\right]
\end{array}},
$$

where $\Lambda_{1}=\rho_{1} /\left(1-\rho_{1}\right), \quad \Lambda_{2}=\rho_{2} /\left(1-\rho_{2}\right), \quad \Omega_{1}=\left(1-\rho_{1}^{l_{1}}\right) /$ $\left(1-\rho_{1}\right), \quad \Omega_{2}=\left(1-\rho_{2}\right) /\left(1-\rho_{2}\right), \quad \Theta_{1}=\left(1-\rho_{1}^{k}\right) /\left(1-\rho_{1}\right)$, $\Theta_{2}=\left(1-\rho_{2}^{k}\right) /\left(1-\rho_{2}\right)$, and $\Delta=(\psi(1-\psi)) /\left(1-\rho_{1} \rho_{2}\right)$.

Proof. Using the MA forecasting technique, the estimate of the standard deviation of the $l_{1}$ period forecast error of R1 $\widehat{\xi}_{1, t}^{l_{1}}$ is a constant and can be expressed as follows:

$$
\begin{aligned}
\hat{\xi}_{1, t}^{l_{1}} & =\sqrt{\operatorname{Var}\left(\hat{d}_{1, t}^{l_{1}}-d_{1, t}^{l_{1}}\right)} \\
& =\sqrt{\operatorname{Var}\left(\hat{d}_{1, t}^{l_{1}}\right)+\operatorname{Var}\left(d_{1, t}^{l_{1}}\right)-2 \operatorname{Cov}\left(\hat{d}_{1, t}^{l_{1}}, d_{1, t}^{l_{1}}\right)},
\end{aligned}
$$

$$
\begin{aligned}
\operatorname{Var}\left(d_{1, t}^{l_{1}}\right)= & \operatorname{Var}\left(\sum_{i=0}^{l_{1}-1} d_{1, t+i}\right) \\
= & \sum_{i=0}^{l_{1}-1} \operatorname{Var}\left(d_{1, t+i}\right)+2 \sum_{i=0}^{l_{1}-2} \sum_{j=1}^{l_{1}-1-i} \operatorname{Cov}\left(d_{1, t+i}, d_{1, t+i+j}\right) \\
= & l_{1}\left(\delta_{1, d}\right)^{2}+2 \sum_{i=0}^{l_{1}-2} \sum_{j=1}^{l_{1}-1-i} \operatorname{Cov}\left(a_{1}-b_{11} p_{1, t+i}+b_{12} p_{2, t+i}+\varepsilon_{1, t+i}, a_{1}-b_{11} p_{1, t+i+j}+b_{12} p_{2, t+i+j}+\varepsilon_{1, t+i+j}\right) \\
= & l_{1}\left(\delta_{1, d}\right)^{2}+2 b_{11}^{2}\left(\delta_{1, p}\right)^{2}\left(\frac{\rho_{1}}{1-\rho_{1}}\right)\left(l_{1}-\frac{1-\rho_{1}^{l_{1}}}{1-\rho_{1}}\right)-2 b_{11} b_{12}\left(\frac{\delta_{12}^{2} \psi(1-\psi)}{1-\rho_{1} \rho_{2}}\right)\left(\frac{\rho_{1}}{1-\rho_{1}}\right)\left(l_{1}-\frac{1-\rho_{1}^{l_{1}}}{1-\rho_{1}}\right) \\
& -2 b_{12} b_{11}\left(\frac{\delta_{12}^{2} \psi(1-\psi)}{1-\rho_{1} \rho_{2}}\right)\left(\frac{\rho_{2}}{1-\rho_{2}}\right)\left(l_{2}-\frac{1-\rho_{2}^{l_{2}}}{1-\rho_{2}}\right)+2 b_{12}^{2}\left(\delta_{2, p}\right)^{2}\left(\frac{\rho_{2}}{1-\rho_{2}}\right)\left(l_{2}-\frac{1-\rho_{2}^{l_{2}}}{1-\rho_{2}}\right),
\end{aligned}
$$




$$
\begin{aligned}
& \operatorname{Var}\left(\begin{array}{c}
\wedge^{l_{1}} \\
d_{1, t}
\end{array}\right)=\left(\frac{l_{1}}{k}\right)^{2} \operatorname{Var}\left(\sum_{i=1}^{k} d_{1, t-i}\right) \\
& =\left(\frac{l_{1}}{k}\right)^{2}\left[k\left(\delta_{1, d}\right)^{2}+2 \operatorname{Cov}\left(d_{1, t-1}, d_{1, t-2}\right)+2 \operatorname{Cov}\left(d_{1, t-1}, d_{1, t-3}\right)+\cdots+2 \operatorname{Cov}\left(d_{1, t-k-1}, d_{1, t-k}\right)\right] \\
& =\left(\frac{l_{1}}{k}\right)^{2}\left[k\left(\delta_{1, d}\right)^{2}+2 b_{11}^{2}\left(\delta_{1, p}\right)^{2}\left(\frac{\rho_{1}}{1-\rho_{1}}\right)\left(k-\frac{1-\rho_{1}^{k}}{1-\rho_{1}}\right)+2 b_{12}^{2}\left(\delta_{2, p}\right)^{2}\left(\frac{\rho_{2}}{1-\rho_{2}}\right)\left(k-\frac{1-\rho_{2}^{k}}{1-\rho_{2}}\right)\right. \\
& \left.-2 b_{11} b_{12}\left(\left(\frac{\rho_{1}}{1-\rho_{1}}\right)\left(k-\frac{1-\rho_{1}^{k}}{1-\rho_{1}}\right)+\left(\frac{\rho_{2}}{1-\rho_{2}}\right)\left(k-\frac{1-\rho_{2}^{k}}{1-\rho_{2}}\right)\right) \frac{\psi(1-\psi)}{1-\rho_{1} \rho_{2}} \delta_{12}^{2}\right], \\
& \operatorname{Cov}\left(d_{1, t}^{l_{1}}, \hat{d}_{1, t}^{l_{1}}\right)=\operatorname{Cov}\left(\sum_{i=0}^{l_{1}-1} d_{1, t+i}, \frac{l_{1}}{k} \sum_{j=1}^{k} d_{1, t-j}\right) \\
& =\frac{l_{1}}{k} \sum_{i=0}^{l_{1}-1} \sum_{j=1}^{k} \operatorname{Cov}\left(d_{1, t+i}, d_{1, t-j}\right) \\
& =\frac{l_{1}}{k} \sum_{i=0}^{l_{1}-1} \sum_{j=1}^{k} \operatorname{Cov}\left(-b_{11} p_{1, t+i}+b_{12} p_{2, t+i},-b_{11} p_{1, t-j}+b_{12} p_{2, t-j}\right) \\
& =\frac{l_{1}}{k} \sum_{i=0}^{l_{1}-1} \sum_{j=1}^{k}\left\{b_{11}^{2} \operatorname{Cov}\left(p_{1, t+i}, p_{1, t-j}\right)-b_{11} b_{12} \operatorname{Cov}\left(p_{1, t+i}, p_{2, t-j}\right)-b_{12} b_{11} \operatorname{Cov}\left(p_{2, t+i}, p_{1, t-j}\right)+b_{12}^{2} \operatorname{Cov}\left(p_{2, t+i}, p_{2, t-j}\right)\right\} \\
& =\frac{l_{1}}{k}\left\{\frac{b_{11}^{2} \rho_{1}\left(1-\rho_{1}^{l_{1}}\right)\left(1-\rho_{1}^{k}\right)\left(\delta_{1, p}\right)^{2}}{\left(1-\rho_{1}\right)^{2}}-b_{11} b_{12} \frac{\delta_{12}^{2} \psi(1-\psi)}{1-\rho_{1} \rho_{2}} \frac{\rho_{1}\left(1-\rho_{1}^{l_{1}}\right)\left(1-\rho_{1}^{k}\right)}{\left(1-\rho_{1}\right)^{2}}\right. \\
& \left.-b_{12} b_{11} \frac{\delta_{12}^{2} \psi(1-\psi)}{1-\rho_{1} \rho_{2}} \frac{\rho_{2}\left(1-\rho_{2}^{l_{2}}\right)\left(1-\rho_{2}^{k}\right)}{\left(1-\rho_{2}\right)^{2}}+\frac{b_{12}^{2} \rho_{2}\left(1-\rho_{2}^{l_{2}}\right)\left(1-\rho_{2}^{k}\right)\left(\delta_{2, p}\right)^{2}}{\left(1-\rho_{2}\right)^{2}}\right\} \text {. }
\end{aligned}
$$

Lemma 2. Using the MA forecasting technique, the estimate of the standard deviation of the $l_{2}$ period forecast error of $R 2 \widehat{\xi}_{2, t}^{l_{2}}$ is a constant and can be expressed as follows:

$$
\hat{\xi}_{2, t}^{l_{2}}=\sqrt{\begin{array}{l}
l_{2} \delta_{2, d}^{2}+2 b_{21}^{2} \delta_{2, p}^{2} \Lambda_{2}\left(l_{2}-\Omega_{2}\right)-2 b_{21} b_{22} \delta_{12}^{2} \Delta \Lambda_{2}\left(l_{2}-\Omega_{2}\right)-2 b_{22} b_{21} \delta_{12}^{2} \Delta \Lambda_{1}\left(l_{1}-\Omega_{1}\right)+2 b_{22}^{2} \delta_{1, p}^{2} \Lambda_{1}\left(l_{1}-\Omega_{1}\right) \\
-2 \frac{l_{2}}{k}\left\{b_{21}^{2} \rho_{2} \Omega_{2} \delta_{2, p}^{2} \Lambda_{2} \delta_{2, p}^{2}-b_{21} b_{22} \delta_{12}^{2} \Delta \rho_{2} \Omega_{2} \Theta_{2}-b_{22} b_{21} \delta_{12}^{2} \Delta \rho_{1} \Omega_{1} \Theta_{1}+b_{22}^{2} \delta_{1, p}^{2} \Lambda_{1} \delta_{1, p}^{2} \Omega_{1} \Theta_{1}\right\},
\end{array}}
$$

where $\Lambda_{1}=\rho_{1} /\left(1-\rho_{1}\right), \Lambda_{2}=\rho_{2} /\left(1-\rho_{2}\right), \quad \Omega_{1}=\left(1-\rho_{1}^{l_{1}}\right) /$ $\left(1-\rho_{1}\right), \quad \Omega_{2}=\left(1-\rho_{2}\right) /\left(1-\rho_{2}\right), \quad \Theta_{1}=\left(1-\rho_{1}^{k}\right) /\left(1-\rho_{1}\right)$, $\Theta_{2}=\left(1-\rho_{2}^{k}\right) /\left(1-\rho_{2}\right)$, and $\Delta=(\psi(1-\psi)) /\left(1-\rho_{1} \rho_{2}\right)$.
Proof. Using the MA forecasting technique, the estimate of the standard deviation of the $l_{2}$ period forecast error of retailer-2 $\widehat{\xi}_{2, t}^{2}$ is a constant and can be expressed as follows: 


$$
\begin{aligned}
\hat{\xi}_{2, t}^{l_{2}} & =\sqrt{\operatorname{Var}\left(\hat{d}_{2, t}^{l_{2}}-d_{2, t}^{l_{2}}\right)} \\
& =\sqrt{\operatorname{Var}\left(\hat{d}_{2, t}^{l_{2}}\right)+\operatorname{Var}\left(d_{2, t}^{l_{2}}\right)-2 \operatorname{Cov}\left(\hat{d}_{2, t}^{l_{2}}, d_{2, t}^{l_{2}}\right)},
\end{aligned}
$$

where

$$
\begin{aligned}
& \operatorname{Var}\left(d_{2, t}^{l_{2}}\right)=\operatorname{Var}\left(\sum_{i=0}^{l_{2}-1} d_{2, t+i}\right)=\sum_{i=0}^{l_{2}-1} \operatorname{Var}\left(d_{2, t+i}\right)+2 \sum_{i=0}^{l_{2}-2} \sum_{j=1}^{l_{2}-1-i} \operatorname{Cov}\left(d_{2, t+i}, d_{2, t+i+j}\right)
\end{aligned}
$$

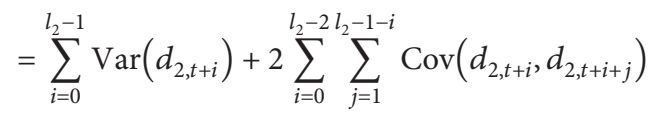

$$
\begin{aligned}
& =l_{2}\left(\delta_{2, d}\right)^{2}+2 \sum_{i=0}^{l_{2}-2} \sum_{j=1}^{l_{2}-1-i} \operatorname{Cov}\left(a_{2}-b_{21} p_{2, t+i}+b_{22} p_{1, t+i}+\varepsilon_{2, t+i}, a_{2}-b_{21} p_{2, t+i+j}+b_{22} p_{1, t+i+j}+\varepsilon_{2, t+i+j}\right) \\
& =l_{2}\left(\delta_{2, d}\right)^{2}+2 b_{21}^{2}\left(\delta_{2, p}\right)^{2}\left(\frac{\rho_{2}}{1-\rho_{2}}\right)\left(l_{2}-\frac{1-\rho_{2}^{l_{2}}}{1-\rho_{2}}\right)-2 b_{21} b_{22}\left(\frac{\delta_{12}^{2} \psi(1-\psi)}{1-\rho_{1} \rho_{2}}\right)\left(\frac{\rho_{2}}{1-\rho_{2}}\right)\left(l_{2}-\frac{1-\rho_{2}^{l_{2}}}{1-\rho_{2}}\right) \\
& -2 b_{22} b_{21}\left(\frac{\delta_{12}^{2} \psi(1-\psi)}{1-\rho_{1} \rho_{2}}\right)\left(\frac{\rho_{1}}{1-\rho_{1}}\right)\left(l_{1}-\frac{1-\rho_{1}^{l_{1}}}{1-\rho_{1}}\right)+2 b_{22}^{2}\left(\delta_{1, p}\right)^{2}\left(\frac{\rho_{1}}{1-\rho_{1}}\right)\left(l_{1}-\frac{1-\rho_{1}^{l_{1}}}{1-\rho_{1}}\right), \\
& \operatorname{Var}\left(\hat{d}_{2, t}^{l_{2}}\right)=\left(\frac{l_{2}}{k}\right)^{2} \operatorname{Var}\left(\sum_{i=1}^{k} d_{2, t-i}\right)=\left(\frac{l_{2}}{k}\right)^{2} \operatorname{Var}\left(d_{2, t-1}+d_{2, t-2}+\cdots+d_{2, t-k}\right) \\
& =\left(\frac{l_{2}}{k}\right)^{2}\left[k\left(\delta_{2, d}\right)^{2}+2 \operatorname{Cov}\left(d_{2, t-1}, d_{2, t-2}\right)+2 \operatorname{Cov}\left(d_{2, t-1}, d_{2, t-3}\right)+\cdots+2 \operatorname{Cov}\left(d_{2, t-k-1}, d_{2, t-k}\right)\right] \\
& =\left(\frac{l_{2}}{k}\right)^{2}\left[k\left(\delta_{2, d}\right)^{2}+2 b_{21}^{2}\left(\delta_{2, p}\right)^{2}\left(\frac{\rho_{2}}{1-\rho_{2}}\right)\left(k-\frac{1-\rho_{2}^{k}}{1-\rho_{2}}\right)+2 b_{22}^{2}\left(\delta_{1, p}\right)^{2}\left(\frac{\rho_{1}}{1-\rho_{1}}\right)\left(k-\frac{1-\rho_{1}^{k}}{1-\rho_{1}}\right)\right. \\
& \left.-2 b_{21} b_{22}\left(\left(\frac{\rho_{1}}{1-\rho_{1}}\right)\left(k-\frac{1-\rho_{1}^{k}}{1-\rho_{1}}\right)+\left(\frac{\rho_{2}}{1-\rho_{2}}\right)\left(k-\frac{1-\rho_{2}^{k}}{1-\rho_{2}}\right)\right) \frac{\psi(1-\psi)}{1-\rho_{1} \rho_{2}} \delta_{12}^{2}\right] \text {, } \\
& \operatorname{Cov}\left(d_{2, t}^{l_{2}}, \hat{d}_{2, t}^{l_{2}}\right)=\operatorname{Cov}\left(\sum_{i=0}^{l_{2}-1} d_{2, t+i}, \frac{l_{2}}{k} \sum_{j=1}^{k} d_{2, t-j}\right)=\frac{l_{2}}{k} \operatorname{Cov}\left(\sum_{i=0}^{l_{2}-1} d_{2, t+i}, \frac{l_{2}}{k} \sum_{j=1}^{k} d_{2, t-j}\right) \\
& =\frac{l_{2}}{k} \sum_{i=0}^{l_{2}-1} \sum_{j=1}^{k} \operatorname{Cov}\left(d_{2, t+i}, d_{2, t-j}\right) \\
& =\frac{l_{2}}{k} \sum_{i=0}^{l_{2}-1} \sum_{j=1}^{k} \operatorname{Cov}\left(-b_{21} p_{2, t+i}+b_{22} p_{1, t+i},-b_{21} p_{2, t-j}+b_{22} p_{1, t-j}\right) \\
& =\frac{l_{2}}{k} \sum_{i=0}^{l_{2}-1} \sum_{j=1}^{k}\left\{b_{21}^{2} \operatorname{Cov}\left(p_{2, t+i}, p_{2, t-j}\right)-b_{21} b_{22} \operatorname{Cov}\left(p_{2, t+i}, p_{1, t-j}\right)-b_{22} b_{21} \operatorname{Cov}\left(p_{1, t+i}, p_{2, t-j}\right)+b_{22}^{2} \operatorname{Cov}\left(p_{1, t+i}, p_{1, t-j}\right)\right\} \\
& =\frac{l_{2}}{k}\left\{\frac{b_{21}^{2} \rho_{2}\left(1-\rho_{2}^{l_{2}}\right)\left(1-\rho_{2}^{k}\right)\left(\delta_{2, p}\right)^{2}}{\left(1-\rho_{2}\right)^{2}}-b_{21} b_{22} \frac{\delta_{12}^{2} \psi(1-\psi)}{1-\rho_{1} \rho_{2}} \frac{\rho_{2}\left(1-\rho_{2}^{l_{2}}\right)\left(1-\rho_{2}^{k}\right)}{\left(1-\rho_{2}\right)^{2}}\right. \\
& \left.-b_{22} b_{21} \frac{\delta_{12}^{2} \psi(1-\psi)}{1-\rho_{1} \rho_{2}} \frac{\rho_{1}\left(1-\rho_{1}^{l_{1}}\right)\left(1-\rho_{1}^{k}\right)}{\left(1-\rho_{1}\right)^{2}}+\frac{b_{22}^{2} \rho_{1}\left(1-\rho_{1}^{l_{1}}\right)\left(1-\rho_{1}^{k}\right)\left(\delta_{1, p}\right)^{2}}{\left(1-\rho_{1}\right)^{2}}\right\} \text {. }
\end{aligned}
$$


Theorem 2. For two parallel supply chains in which there is a competition effect on customer demand and the retailers' market share, when two retailers use the order-up-to inventory strategy as well as the MA forecasting technique, the quantitative model of the inventory bullwhip effect in two supply chains is expressed as follows:

$$
\begin{gathered}
\mathrm{IBE}_{\text {retailer-1 }}^{\mathrm{MA}}=\frac{\operatorname{Var}\left(I_{1, t}\right)}{\operatorname{Var}\left(d_{1, t}\right)}=\frac{\left\{\begin{array}{c}
l_{1} \delta_{1, d}^{2}+2 b_{11}^{2} \delta_{1, p}^{2} \Lambda_{1}\left(l_{1}-\Omega_{1}\right)-2 b_{11} b_{12} \delta_{12}^{2} \Delta \Lambda_{1}\left(l_{1}-\Omega_{1}\right)-2 b_{12} b_{11} \delta_{12}^{2} \Delta \Lambda_{2}\left(l_{2}-\Omega_{2}\right)+2 b_{12}^{2} \delta_{2, p}^{2} \Lambda_{2}\left(l_{2}-\Omega_{2}\right) \\
+\left(l_{1} / k\right)^{2}\left[k \delta_{1, d}^{2}+2 b_{11}^{2} \delta_{1, p}^{2} \Lambda_{1}\left(k-\Theta_{1}\right)+2 b_{12}^{2} \delta_{2, p}^{2} \Lambda_{2}\left(k-\Theta_{2}\right)-2 b_{11} b_{12}\left(\Lambda_{1}\left(k-\Theta_{1}\right)+\Lambda_{2}\left(k-\Theta_{2}\right)\right) \Delta \delta_{12}^{2}\right] \\
-2 l_{1} / k\left\{b_{11} b_{21} \rho_{1} \Omega_{1} \Theta_{1} \delta_{1, p}^{2}-b_{11} b_{22} \rho_{1} \Omega_{1} \Theta_{1} \delta_{12}^{2} \Delta-b_{12} b_{21} \rho_{2} \Omega_{2} \Theta_{2} \Delta \delta_{12}^{2}+b_{12} b_{22} \rho_{2} \Omega_{2} \Theta_{2} \delta_{2, p}^{2}\right\}
\end{array}\right\}}{\delta_{1, d}^{2}}, \\
\mathrm{IBE}_{\text {retailer-2 }}^{\mathrm{MA}}=\frac{\operatorname{Var}\left(I_{2, t}\right)}{\operatorname{Var}\left(d_{2, t}\right)}=\frac{\left\{\begin{array}{c}
l_{2} \delta_{2, d}^{2}+2 b_{21}^{2} \delta_{2, p}^{2} \Lambda_{2}\left(l_{2}-\Omega_{2}\right)-2 b_{21} b_{22} \delta_{12}^{2} \Delta \Lambda_{2}\left(l_{2}-\Omega_{2}\right)-2 b_{22} b_{21} \delta_{12}^{2} \Delta \Lambda_{1}\left(l_{1}-\Omega_{1}\right)+2 b_{22}^{2} \delta_{1, p}^{2} \Lambda_{1}\left(l_{1}-\Omega_{1}\right) \\
+\left(l_{2} / k\right)^{2}\left[k \delta_{2, d}^{2}+2 b_{21}^{2} \delta_{2, p}^{2} \Lambda_{2}\left(k-\Theta_{2}\right)+2 b_{22}^{2} \delta_{1, p}^{2} \Lambda_{1}\left(k-\Theta_{1}\right)-2 b_{21} b_{22}\left(\Lambda_{1}\left(k-\Theta_{1}\right)+\Lambda_{2}\left(k-\Theta_{2}\right)\right) \Delta \delta_{12}^{2}\right] \\
-2 l_{2} / k\left\{b_{21}^{2} \rho_{2} \Omega_{2} \Theta_{2} \delta_{2, p}^{2}-b_{21} b_{22} \delta_{12}^{2} \Delta \rho_{2} \Omega_{2} \Theta_{2}-b_{22} b_{21} \delta_{12}^{2} \Delta \rho_{1} \Omega_{1} \Theta_{1}+b_{22}^{2} \rho_{1} \delta_{1, p}^{2} \Omega_{1} \Theta_{1}\right\}
\end{array}\right.}{\delta_{2, d}^{2}},
\end{gathered}
$$

where $\Lambda_{1}=\rho_{1} /\left(1-\rho_{1}\right), \quad \Lambda_{2}=\rho_{2} /\left(1-\rho_{2}\right), \quad \Omega_{1}=\left(1-\rho_{1}^{l_{1}}\right) /$ $\left(1-\rho_{1}\right), \quad \Omega_{2}=\left(1-\rho_{2}^{2}\right) /\left(1-\rho_{2}\right), \quad \Theta_{1}=\left(1-\rho_{1}^{k}\right) /\left(1-\rho_{1}\right)$, $\Theta_{2}=\left(1-\rho_{2}^{k}\right) /\left(1-\rho_{2}\right)$, and $\Delta=(\psi(1-\psi)) /\left(1-\rho_{1} \rho_{2}\right)$.

5.2. The Inventory Bullwhip Effect Using the ES Method. In equation (6), we can obtain the mean lead-time demands $\hat{d}_{1, t} l_{1}$ and $\hat{d}_{2, t}^{2}$ using the exponential smoothing forecasting technique, where $\alpha(0<\alpha<1)$ is a smoothing constant for the two retailers:

$$
\begin{aligned}
& \hat{d}_{1, t}^{l_{1}}=l_{1}\left[\alpha d_{1, t-1}+(1-\alpha) \hat{d}_{1, t-1}\right], \\
& \hat{d}_{2, t}^{l_{2}}=l_{2}\left[\alpha d_{2, t-1}+(1-\alpha) \hat{d}_{2, t-1}\right] .
\end{aligned}
$$

Then, $q_{1, t}$ and $q_{2, t}$ in equation (6) can be written as the following equation:

$$
\begin{aligned}
& q_{1, t}=\left(1+\alpha l_{1}\right) d_{1, t-1}-\alpha l_{1} \widehat{d}_{1, t-1}+z_{1}\left(\widehat{\xi}_{1, t}^{l_{1}}-\widehat{\xi}_{1, t-1}^{l_{1}}\right), \\
& q_{2, t}=\left(1+\alpha l_{2}\right) d_{2, t-1}-\alpha l_{2} \widehat{d}_{2, t-1}+z_{2}\left(\widehat{\xi}_{2, t}^{l_{2}}-\widehat{\xi}_{2, t-1}^{l_{2}}\right) .
\end{aligned}
$$

Theorem 3. For two parallel supply chains in which there is a competition effect on customer demand and the retailers' market share, when two retailers use the order-up-to inventory strategy as well as the ES forecasting technique, the quantitative model of the bullwhip effect in two supply chains is expressed as follows:

$$
\begin{aligned}
\mathrm{BE}_{\text {retailer-1 }}^{\mathrm{ES}} & =\frac{\operatorname{Var}\left(q_{1, t}\right)}{\operatorname{Var}\left(d_{1, t}\right)} \\
& =\frac{\left(1+\alpha l_{1}\right)^{2} \delta_{1, d}^{2}+\alpha^{2} l_{1}^{2}(\alpha / 2-\alpha) \delta_{1, d}^{2}+\left(\alpha^{2} l_{1}^{2}((2(1-\alpha)) /(2-\alpha))-2 \alpha l_{1}\left(1+\alpha l_{1}\right)\right)\left[\left(\psi \alpha b_{11}^{2} \rho_{1} \delta_{1, p}^{2}\right) /\left(\left(1-\rho_{1}^{2}\right)\left(1-(1-\alpha) \rho_{1}\right)\right) n+q\left((1-\psi) \alpha b_{12}^{2} \rho_{2} \delta_{2, p}^{2}\right) /\left(\left(1-\rho_{2}^{2}\right)\left(1-(1-\alpha) \rho_{2}\right)\right)\right]}{\delta_{1, d}^{2}}, \\
\mathrm{BE}_{\text {retailer-2 }}^{\mathrm{ES}} & =\frac{\operatorname{Var}\left(q_{2, t}\right)}{\operatorname{Var}\left(d_{2, t}\right)} \\
& =\frac{\left(1+\alpha l_{2}\right)^{2} \delta_{2, d}^{2}+\alpha^{2} l_{2}^{2}(\alpha / 2-\alpha) \delta_{2, d}^{2}+\left(\alpha^{2} l_{2}^{2}((2(1-\alpha)) /(2-\alpha))-2 \alpha l_{2}\left(1+\alpha l_{2}\right)\right)\left[\left(\psi \alpha b_{21}^{2} \rho_{2} \delta_{2, p}^{2}\right) /\left(\left(1-\rho_{2}^{2}\right)\left(1-(1-\alpha) \rho_{2}\right)\right) n+q\left((1-\psi) \alpha b_{22}^{2} \rho_{1} \delta_{1, p}^{2}\right) /\left(\left(1-\rho_{1}^{2}\right)\left(1-(1-\alpha) \rho_{1}\right)\right)\right]}{\delta_{2, d}^{2}} .
\end{aligned}
$$

Similar to Section 5.1, in order to analyze the impact of the bullwhip effect on the inventory level, we should calculate the variance of the inventory level for the two retailers at period $t$ as $I_{1, t}$ and $I_{2, t}$ corresponding to the bullwhip effect. $I_{1, t}$ and $I_{2, t}$ can be expressed as follows:

$$
\begin{aligned}
& I_{1, t}=I_{1, t-1}+q_{1, t-l_{1}}-d_{1, t-1}, \\
& I_{2, t}=I_{2, t-1}+q_{2, t-l_{2}}-d_{2, t-1} .
\end{aligned}
$$

From equation (25), we can determine that

$$
\begin{aligned}
& q_{1, t-l_{1}}=I_{1, t}-I_{1, t-1}+d_{1, t-1}, \\
& q_{2, t-l_{2}}=I_{2, t}-I_{2, t-1}+d_{2, t-1} .
\end{aligned}
$$

Vassian [48] proposed that $q_{1, t-l_{1}}$ and $q_{2, t-l_{2}}$ can be expressed as follows: 


$$
\begin{aligned}
& q_{1, t-l_{1}}=\hat{d}_{1, t}^{l_{1}}-\sum_{i=1}^{l_{1}-1} q_{1, t-i}-I_{1, t}, \\
& q_{2, t-l_{2}}=\widehat{d}_{2, t}^{l_{2}}-\sum_{i=1}^{l_{2}-1} q_{2, t-i}-I_{2, t} .
\end{aligned}
$$

We substituted equation (27) into equation (26), and we can obtain equation (28) as follows:

$$
\begin{aligned}
& I_{1, t}=\widehat{\xi}_{1, t}^{l_{1}}=\hat{d}_{1, t-l_{1}}^{l_{1}}-d_{1, t-l_{1}}^{l_{1}}, \\
& I_{2, t}=\widehat{\xi}_{2, t}^{l_{2}}=\hat{d}_{2, t-l_{2}}^{l_{2}}-d_{2, t-l_{2}}^{l_{2}} .
\end{aligned}
$$

Lemma 3. Using the ES forecasting technique, the estimate of the standard deviation of the $l_{1}$ period forecast error of $R 1 \widehat{\xi}_{1, t}^{1}$ is a constant and can be expressed as follows:

$$
\hat{\xi}_{1, t}^{l_{1}}=\sqrt{\left(l_{1}+l_{1}^{2} \frac{\alpha}{2-\alpha}\right) \delta_{1, d}^{2}+2 \psi b_{11}^{2} \rho_{1}\left(\frac{\left(l_{1}-\left(1-\rho_{1}^{l_{1}}\right)\right)}{\left(1-\rho_{1}\right)^{2}}+\frac{l_{1}^{2}(1-\alpha) \alpha}{(2-\alpha)\left(1-(1-\alpha) \rho_{1}\right)}-\frac{l_{1} \alpha\left(1-\rho_{1}^{l_{1}}\right)}{\left(1-\rho_{1}\right)\left(1-(1-\alpha) \rho_{1}\right)}\right) \delta_{1, p}^{2}+2(1-\psi) b_{12}^{2} \rho_{2}\left(\frac{\left(l_{2}-\left(1-\rho_{2}^{l_{2}}\right)\right)}{\left(1-\rho_{2}\right)^{2}}+\frac{l_{1}^{2}(1-\alpha) \alpha}{(2-\alpha)\left(1-(1-\alpha) \rho_{2}\right)}-\frac{l_{1} \alpha\left(1-\rho_{2}^{l_{1}}\right)}{\left(1-\rho_{2}\right)\left(1-(1-\alpha) \rho_{2}\right)}\right) \delta_{2, p}^{2}} .
$$

Proof. Using the ES forecasting technique, the estimate of the standard deviation of the $l_{1}$ period forecast error of R1 $\widehat{\xi}_{1, t}^{l_{1}}$ is a constant and can be expressed as follows:

$$
\widehat{\xi}_{1, t}^{l_{1}}=\sqrt{\operatorname{Var}\left(d_{1, t}^{l_{1}}\right)+\operatorname{Var}\left(\hat{d}_{1, t}^{l_{1}}\right)-2 \operatorname{Cov}\left(d_{1, t}^{l_{1}}, \widehat{d}_{1, t}^{l_{1}}\right)},
$$

where

$$
\begin{aligned}
\operatorname{Var}\left(d_{1, t}^{l_{1}}\right) & =\operatorname{Var}\left(l_{1}\left(a_{1}-b_{11} p_{1, t}+b_{12} p_{2, t}+\varepsilon_{1, t}\right)\right) \\
& =l_{1} \delta_{1, d}^{2}+\frac{2 \psi b_{11}^{2} \rho_{1}\left(l_{1}-\left(1-\rho_{1}^{l_{1}}\right)\right) \delta_{1, p}^{2}}{\left(1-\rho_{1}\right)^{2}}+\frac{2(1-\psi) b_{12}^{2} \rho_{2}\left(l_{2}-\left(1-\rho_{2}^{l_{2}}\right)\right) \delta_{2, p}^{2}}{\left(1-\rho_{2}\right)^{2}} \\
\operatorname{Var}\left(\hat{d}_{1, t}^{l_{1}}\right) & =l_{1}^{2} \operatorname{Var}\left(\hat{d}_{1, t}\right) \\
& =l_{1}^{2}\left[\frac{\alpha}{2-\alpha} \operatorname{Var}\left(d_{1, t}\right)+\frac{2(1-\alpha)}{2-\alpha} \operatorname{Cov}\left(d_{1, t-1}, \widehat{d}_{1, t}\right)\right] \\
& =l_{1}^{2}\left[\frac{\alpha}{2-\alpha} \delta_{1, d}^{2}+\frac{2 \psi(1-\alpha) \alpha b_{11}^{2} \rho_{1}}{(2-\alpha)\left(1-(1-\alpha) \rho_{1}\right)} \delta_{1, p}^{2}+\frac{2(1-\psi)(1-\alpha) \alpha b_{12}^{2} \rho_{2}}{(2-\alpha)\left(1-(1-\alpha) \rho_{2}\right)} \delta_{2, p}^{2}\right] \\
\operatorname{Cov}\left(d_{1, t}^{l_{1}}, \hat{d}_{1, t}^{l_{1}}\right) & =\operatorname{Cov}\left(\sum_{i=0}^{l_{1}-1}\left(d_{1, t+i}, l_{1} \hat{d}_{1, t}\right)\right)=l_{1} \sum_{i=0}^{l_{1}-1} \operatorname{Cov}\left(d_{1, t+i}, \hat{d}_{1, t}\right) \\
& =\frac{\psi l_{1} \alpha b_{11}^{2} \rho_{1}\left(1-\rho_{1}^{l_{1}}\right)}{\left(1-\rho_{1}\right)\left(1-(1-\alpha) \rho_{1}\right)} \delta_{1, p}^{2}+\frac{(1-\psi) l_{1} \alpha b_{12}^{2} \rho_{2}\left(1-\rho_{2}^{l_{1}}\right)}{\left(1-\rho_{2}\right)\left(1-(1-\alpha) \rho_{2}\right)} \delta_{2, p}^{2} . \\
& l_{1} \psi \alpha b_{11}^{2} \sum_{i=0}^{l_{1}-1} \sum_{j=1}^{\infty}(1-\alpha)^{j-1} \rho_{1}^{i+j} \delta_{1, p}^{2}+(1-\psi) l_{1} \alpha b_{12}^{2} \sum_{i=0}^{l_{1}-1} \sum_{j=1}^{\infty}(1-\alpha)^{j-1} \rho_{2}^{i+j} \delta_{2, p}^{2} \\
& \\
& \\
&
\end{aligned}
$$

Lemma 4. Using the ES forecasting technique, the estimate of the standard deviation of the $l_{2}$ period forecast error of $R 2 \widehat{\xi}_{2, t}^{2}$ is a constant and can be expressed as follows:

$$
\hat{\xi}_{2, t}^{2}=\sqrt{\left(l_{2}+l_{2}^{2} \frac{\alpha}{2-\alpha}\right) \delta_{2, d}^{2}+2 \psi b_{21}^{2} \rho_{2}\left(\frac{\left(l_{2}-\left(1-\rho_{2}^{l_{2}}\right)\right)}{\left(1-\rho_{2}\right)^{2}}+\frac{l_{2}^{2}(1-\alpha) \alpha}{(2-\alpha)\left(1-(1-\alpha) \rho_{2}\right)}-\frac{l_{2} \alpha\left(1-\rho_{2}^{l_{2}}\right)}{\left(1-\rho_{2}\right)\left(1-(1-\alpha) \rho_{2}\right)}\right) \delta_{2, p}^{2}+2(1-\psi) b_{22}^{2} \rho_{1}\left(\frac{\left(l_{2}-\left(1-\rho_{1}^{l_{2}}\right)\right)}{\left(1-\rho_{1}\right)^{2}}+\frac{l_{2}^{2}(1-\alpha) \alpha}{(2-\alpha)\left(1-(1-\alpha) \rho_{1}\right)}-\frac{l_{2} \alpha\left(1-\rho_{1}^{l_{1}}\right)}{\left(1-\rho_{1}\right)\left(1-(1-\alpha) \rho_{1}\right)}\right) \delta_{1, p}^{2} .}
$$


Proof. Using the ES forecasting technique, the estimate of the standard deviation of the $l_{2}$ period forecast error of R2 $\widehat{\xi}_{2, t}^{2}$ is a constant and can be expressed as follows:

$$
\widehat{\xi}_{2, t}^{l_{2}}=\sqrt{\operatorname{Var}\left(d_{2, t}^{l_{2}}\right)+\operatorname{Var}\left(\hat{d}_{2, t}^{l_{2}}\right)-2 \operatorname{Cov}\left(d_{2, t}^{l_{2}}, \widehat{d}_{2, t}^{l_{2}}\right)}
$$

where

$$
\begin{aligned}
& \operatorname{Var}\left(d_{2, t}^{l_{2}}\right)=\operatorname{Var}\left(l_{2}\left(a_{2}-b_{21} p_{2, t}+b_{22} p_{1, t}+\varepsilon_{2, t}\right)\right) \\
& =l_{2} \delta_{2, d}^{2}+\frac{2(1-\psi) b_{21}^{2} \rho_{2}\left(l_{2}-\left(1-\rho_{2}^{l_{2}}\right)\right) \delta_{2, p}^{2}}{\left(1-\rho_{2}\right)^{2}}+\frac{2 \psi b_{22}^{2} \rho_{1}\left(l_{1}-\left(1-\rho_{1}^{l_{1}}\right)\right) \delta_{1, p}^{2}}{\left(1-\rho_{1}\right)^{2}}, \\
& \operatorname{Var}\left(\widehat{d}_{2, t}^{l_{2}}\right)=l_{2}^{2} \operatorname{Var}\left(\widehat{d}_{2, t}\right) \\
& =l_{2}^{2}\left[\frac{\alpha}{2-\alpha} \operatorname{Var}\left(d_{2, t}\right)+\frac{2(1-\alpha)}{2-\alpha} \operatorname{Cov}\left(d_{2, t-1}, \widehat{d}_{2, t}\right)\right] \\
& =l_{2}^{2}\left[\frac{\alpha}{2-\alpha} \delta_{2, d}^{2}+\frac{2(1-\psi)(1-\alpha) \alpha b_{21}^{2} \rho_{2}}{(2-\alpha)\left(1-(1-\alpha) \rho_{2}\right)} \delta_{2, p}^{2}+\frac{2 \psi(1-\alpha) \alpha b_{22}^{2} \rho_{1}}{(2-\alpha)\left(1-(1-\alpha) \rho_{1}\right)} \delta_{1, p}^{2}\right] \text {, } \\
& \operatorname{Cov}\left(d_{2, t}^{l_{2}}, \hat{d}_{2, t}^{l_{2}}\right)=\operatorname{Cov}\left(\sum_{i=0}^{l_{2}-1}\left(d_{2, t+i}, l_{2} \hat{d}_{2, t}\right)\right)=l_{2} \sum_{i=0}^{l_{2}-1} \operatorname{Cov}\left(d_{2, t+i}, \hat{d}_{2, t}\right) \\
& =l_{2}(1-\psi) \alpha b_{21}^{2} \sum_{i=0}^{l_{2}-1} \sum_{j=1}^{\infty}(1-\alpha)^{j-1} \rho_{2}^{i+j} \delta_{2, p}^{2}+\psi l_{2} \alpha b_{22}^{2} \sum_{i=0}^{l_{2}-1} \sum_{j=1}^{\infty}(1-\alpha)^{j-1} \rho_{1}^{i+j} \delta_{1, p}^{2} \\
& =\frac{(1-\psi) l_{2} \alpha b_{21}^{2} \rho_{2}\left(1-\rho_{2}^{l_{2}}\right)}{\left(1-\rho_{2}\right)\left(1-(1-\alpha) \rho_{2}\right)} \delta_{2, p}^{2}+\frac{\psi l_{2} \alpha b_{22}^{2} \rho_{1}\left(1-\rho_{1}^{l_{2}}\right)}{\left(1-\rho_{1}\right)\left(1-(1-\alpha) \rho_{1}\right)} \delta_{1, p}^{2} .
\end{aligned}
$$

Theorem 4. For two parallel supply chains in which interactions exist with respect to customer demand and the retailers' market share, when two retailers use the order-up-to inventory strategy and the ES forecasting method, the quantitative model of the inventory bullwhip effect in two supply chains is expressed as follows:

\footnotetext{
$\operatorname{IBE}_{\text {retalier }-1}^{\mathrm{ES}}=\frac{\operatorname{Var}\left(I_{1, c}\right)}{\operatorname{Var}\left(d_{1, t}\right)}$

$=\frac{\left(l_{1}+l_{1}^{2}(\alpha /(2-\alpha))\right) \delta_{1, d}^{2}+2 \psi b_{11}^{2} \rho_{1}\left(\left(l_{1}-\left(1-\rho_{1}^{l_{1}}\right)\right) /\left(1-\rho_{1}\right)^{2} n+q\left(\left(l_{1}^{2}(1-\alpha) \alpha\right) /\left((2-\alpha)\left(1-(1-\alpha) \rho_{1}\right)\right)\right) h-\left(l_{1} \alpha\left(1-\rho_{1}^{l_{1}}\right)\right) /\left(\left(1-\rho_{1}\right)\left(1-(1-\alpha) \rho_{1}\right)\right)\right) \delta_{1, p}^{2}+2(1-\psi) b_{12}^{2} \rho_{2}\left(\left(l_{2}-\left(1-\rho_{2}^{l}\right)\right)\right) /\left(1-\rho_{2}\right)^{2} n+q\left(l_{1}^{2}(1-\alpha) \alpha\right) /\left((2-\alpha)\left(1-(1-\alpha) \rho_{2}\right)\right) h-\left(\left(l_{1} \alpha\left(1-\rho_{2}^{l}\right)\right) /\left(\left(1-\rho_{2}\right)\left(1-(1-\alpha) \rho_{2}\right)\right)\right) \delta_{2, p}^{2}}{\delta_{1, d}}$, $\operatorname{IBE}_{\text {retaler-2 }-2}^{\mathrm{ES}}=\frac{\operatorname{Var}\left(I_{2, t}\right)}{\operatorname{Var}\left(d_{2, t}\right)}$

5.3. The Inventory Bullwhip Effect Using the MMSE Method. Box and Jenkins [49] have pointed out that the demand forecast value $\widehat{d}_{t+i}$ is the conditional expectation in historical demand information for the period $t+i(i=0,1,2, \ldots)$. $\widehat{d}_{t+i}=E\left(d_{t+i} \mid d_{t-1}, d_{t-2}, \ldots\right)$, and particularly for the $\operatorname{AR}(1)$ process, $\widehat{d}_{t+i}=E\left(d_{t+i} \mid d_{t-1}\right)$. In this paper, the price follows the $\mathrm{AR}(1)$ process, such that $\widehat{p}_{t+i}=E\left(p_{t+i} \mid p_{t-1}\right)$. Thus, the demand forecast values for R1 and R2 can be expressed as $\widehat{d}_{1, t+i}=a_{1}-b_{11} \widehat{p}_{1, t+i}+b_{12} \widehat{p}_{2, t+i}+\varepsilon_{1, t+i}$ and $\widehat{d}_{2, t+i}=a_{2}-$ $b_{21} \widehat{p}_{2, t+i}+b_{22} \widehat{p}_{1, t+i}+\varepsilon_{2, t+i}$ in the period $t+i$. Thus, the price forecast values for the period $t+i$ can be expressed as follows: 


$$
\begin{aligned}
& \widehat{p}_{1, t+i}=E\left(p_{1, t+i} \mid p_{1, t-1}\right)=\frac{1-\rho_{1}^{i+1}}{\rho_{1}} \mu_{1} \psi+\rho_{1}^{i+1} p_{1, t-1} \\
& \widehat{p}_{2, t+i}=E\left(p_{2, t+i} \mid p_{2, t-1}\right)=\frac{1-\rho_{2}^{i+1}}{\rho_{2}} \mu_{2}(1-\psi)+\rho_{2}^{i+1} p_{2, t-1} .
\end{aligned}
$$

Thus, the demand forecast values for the period $t+i$ can be expressed as follows:

$$
\begin{aligned}
\hat{d}_{1, t+i} & =a_{1}-b_{11} \widehat{p}_{1, t+i}+b_{12} \widehat{p}_{2, t+i} \\
& =a_{1}-\left(\frac{b_{11}\left(1-\rho_{1}^{i+1}\right) \mu_{1} \psi}{1-\rho_{1}}-\frac{b_{12}\left(1-\rho_{2}^{i+1}\right) \mu_{2}(1-\psi)}{1-\rho_{2}}\right)-\left(b_{11} \rho_{1}^{i+1} p_{1, t-1}-b_{12} \rho_{2}^{i+1} p_{2, t-1}\right), \\
\widehat{d}_{2, t+i} & =a_{2}-b_{21} \widehat{p}_{2, t+i}+b_{22} \widehat{p}_{1, t+i} \\
& =a_{2}-\left(\frac{b_{21}\left(1-\rho_{2}^{i+1}\right) \mu_{2}(1-\psi)}{1-\rho_{2}}-\frac{b_{22}\left(1-\rho_{1}^{i+1}\right) \mu_{1} \psi}{1-\rho_{1}}\right)-\left(b_{21} \rho_{2}^{i+1} p_{2, t-1}-b_{22} \rho_{1, i+1} p_{1, t-1}\right) .
\end{aligned}
$$

Then, $q_{1, t}$ and $q_{2, t}$ in equation (6) can be formulated as equation (37):

$$
\begin{aligned}
& q_{1, t}=-b_{11} \rho_{1} \frac{\rho_{1} \psi\left(1-\rho_{1}^{l_{1}}\right)\left(p_{1, t-1}-p_{1, t-2}\right)}{1-\rho_{1}}+b_{12} \rho_{2} \frac{\rho_{2}\left(1-\rho_{2}^{l_{1}}\right)\left(p_{2, t-1}-p_{2, t-2}\right)}{1-\rho_{2}}+d_{1, t-1}, \\
& q_{2, t}=-b_{21} \rho_{2} \frac{\rho_{2}(1-\psi)\left(1-\rho_{2}^{l_{2}}\right)\left(p_{2, t-1}-p_{2, t-2}\right)}{1-\rho_{2}}+b_{22} \rho_{1} \frac{\rho_{1}\left(1-\rho_{1}^{l_{2}}\right)\left(p_{1, t-1}-p_{1, t-2}\right)}{1-\rho_{1}}+d_{2, t-1} .
\end{aligned}
$$

Theorem 5. For two parallel supply chains in which there is a competition effect on customer demand and the retailers' market share, when two retailers use the order-up-to inventory strategy and the MMSE forecasting technique, the quantitative model of the bullwhip effect in two supply chains is expressed as follows:

$$
\begin{aligned}
& 2 b_{11}^{2} \psi\left(\left(\rho_{1}^{3}\left(1-\rho_{1}^{l_{1}}\right)\left(1-\rho_{1}^{l_{1}+1}\right)\right) /\left(\left(1+\rho_{1}\right)\left(1-\rho_{1}\right)^{2}\right)\right) \delta_{1}^{2}+2 b_{12}^{2}\left(\left(\rho_{2}^{3}\left(1-\rho_{2}^{l_{1}}\right)\left(1-\rho_{2}^{l_{1}+1}\right)\right) /\left(\left(1+\rho_{2}\right)\left(1-\rho_{2}\right)^{2}\right)\right) \delta_{2}^{2} \\
&-2 b_{11} b_{12}\left(\left(\left(\rho_{1}\left(1-\rho_{2}\right)\right) /\left(1-\rho_{1} \rho_{2}\right)\right)+\rho_{1}\left(\left(\rho_{2}\left(1-\rho_{1}\right)\right) /\left(1-\rho_{1} \rho_{2}\right)\right)\left(\left(\rho_{2}\left(1-\rho_{2}^{l_{1}}\right)\right) /\left(1-\rho_{2}^{2}\right)\right)\right)\left(\left(\rho_{1}\left(1-\rho_{1}^{l_{1}}\right)\right) /\left(1-\rho_{1}^{2}\right)\right) \\
& \mathrm{BE}_{\text {retailer-1 }}^{\mathrm{MMSE}}=\frac{\operatorname{Var}\left(q_{1, t}\right)}{\operatorname{Var}\left(d_{1, t}\right)}=1+\frac{\left.+\left(\left(\left(\rho_{2}\left(1-\rho_{1}\right)\right) /\left(1-\rho_{1} \rho_{2}\right)\right)+\rho_{2}\left(\left(\rho_{1}\left(1-\rho_{2}\right)\right) /\left(1-\rho_{1} \rho_{2}\right)\right)\left(\left(\rho_{1}\left(1-\rho_{1}^{l_{1}}\right)\right) /\left(1-\rho_{1}^{2}\right)\right)\right)\left(\left(\rho_{2}\left(1-\rho_{2}^{l_{1}}\right)\right) /\left(1-\rho_{2}^{2}\right)\right)\right) \delta_{12}^{2}}{\delta_{1, d}^{2}}, \\
&
\end{aligned}
$$

Similar to Section 5.1, in order to analyze the impact of the bullwhip effect on the inventory level, we calculated the variance of the inventory level for the two retailers at the period $t$ as $I_{1, t}$ and $I_{2, t}$ corresponding to the bullwhip effect. $I_{1, t}$ and $I_{2, t}$ can be expressed as follows:

$$
\begin{aligned}
& I_{1, t}=I_{1, t-1}+q_{1, t-l_{1}}-d_{1, t-1}, \\
& I_{2, t}=I_{2, t-1}+q_{2, t-l_{2}}-d_{2, t-1} .
\end{aligned}
$$

From equation (40), we can determine 


$$
\begin{aligned}
& q_{1, t-l_{1}}=I_{1, t}-I_{1, t-1}+d_{1, t-1}, \\
& q_{2, t-l_{2}}=I_{2, t}-I_{2, t-1}+d_{2, t-1} .
\end{aligned}
$$

Vassian [48] proposed that $q_{1, t-l_{1}}$ and $q_{2, t-l_{2}}$ can be expressed as follows:

$$
\begin{aligned}
& q_{1, t-l_{1}}=\hat{d}_{1, t}^{l_{1}}-\sum_{i=1}^{l_{1}-1} q_{1, t-i}-I_{1, t}, \\
& q_{2, t-l_{2}}=\hat{d}_{2, t}^{l_{2}}-\sum_{i=1}^{l_{2}-1} q_{2, t-i}-I_{2, t} .
\end{aligned}
$$

We substituted equation (42) into equation (41), and we can obtain equation (43) as follows:

$$
\begin{aligned}
& I_{1, t}=\hat{\xi}_{1, t}^{l_{1}}=\hat{d}_{1, t-l_{1}}^{l_{1}}-d_{1, t-l_{1}}^{l_{1}}, \\
& I_{2, t}=\widehat{\xi}_{2, t}^{l_{2}}=\hat{d}_{2, t-l_{2}}^{l_{2}}-d_{2, t-l_{2}}^{l_{2}} .
\end{aligned}
$$

Lemma 5. Using the MMSE forecasting technique, the estimate of the standard deviation of the $l_{1}$ period forecast error of $R 1 \widehat{\xi}_{1, t}^{1}$ is a constant and can be expressed as follows:

$$
\widehat{\xi}_{1, t}^{l_{1}}=\sqrt{l_{1} \sigma_{1}^{2}+l_{2} \sigma_{2}^{2}+\frac{b_{11}^{2} \psi^{2}}{\left(1-\rho_{1}\right)^{2}}\left(l_{1}+\frac{\rho_{1}\left(1-\rho_{1}^{l_{1}}\right)\left(\rho_{1}^{l_{1}+1}-\rho_{1}-2\right)}{1-\rho_{1}^{2}}\right) \delta_{1}^{2}+\frac{b_{12}^{2}(1-\psi)^{2}}{\left(1-\rho_{2}\right)^{2}}\left(l_{2}+\frac{\rho_{2}\left(1-\rho_{2}^{l_{2}}\right)\left(\rho_{2}^{l_{2}+1}-\rho_{2}-2\right)}{1-\rho_{2}^{2}}\right) \delta_{2}^{2}} .
$$

Proof. Proof

$$
\begin{aligned}
& \widehat{\xi}_{1, t}^{l_{1}}=\sqrt{\operatorname{Var}\left(\hat{d}_{1, t}^{l_{1}}-d_{1, t}^{l_{1}}\right)} \\
& =\sqrt{\operatorname{Var}\left(\sum_{i=0}^{l_{1}-1}\left(-b_{11} \psi \sum_{j=0}^{i} \rho_{1}^{i-j} \eta_{1, t+j}-b_{12}(1-\psi) \sum_{j=0}^{t} \rho_{2}^{i-j} \eta_{2, t+j}+\varepsilon_{1, t+i}+\varepsilon_{2, t+i}\right)\right)} \\
& =\sqrt{\operatorname{Var}\left(\sum_{i=0}^{l_{1}-1} \varepsilon_{1, t+i}\right)+\operatorname{Var}\left(\sum_{i=0}^{l_{2}-1} \varepsilon_{2, t+i}\right)+b_{11}^{2} \psi^{2} \operatorname{Var}\left(\sum_{i=0}^{l_{1}-1} \sum_{j=0}^{i} \rho_{1}^{i-j} \eta_{1, t+j}\right)+b_{12}^{2}(1-\psi)^{2} \operatorname{Var}\left(\sum_{i=0}^{l_{2}-1} \sum_{j=0}^{i} \rho_{2}^{i-j} \eta_{2, t+j}\right)} \\
& =\sqrt{l_{1} \sigma_{1}^{2}+l_{2} \sigma_{2}^{2}+b_{11}^{2} \psi^{2} \operatorname{Var}\left(\sum_{i=0}^{l_{1}-1} \eta_{1, t+j} \sum_{j=0}^{l_{1}-1-i} \rho_{1}^{j}\right)+b_{12}^{2}(1-\psi)^{2} \operatorname{Var}\left(\sum_{i=0}^{l_{2}-1} \eta_{2, t+j} \sum_{j=0}^{l_{2}-1-i} \rho_{2}^{j}\right)} \\
& =\sqrt{l_{1} \sigma_{1}^{2}+l_{2} \sigma_{2}^{2}+\frac{b_{11}^{2} \psi^{2}}{\left(1-\rho_{1}\right)^{2}}\left(l_{1}+\frac{\rho_{1}\left(1-\rho_{1}^{l_{1}}\right)\left(\rho_{1}^{l_{1}+1}-\rho_{1}-2\right)}{1-\rho_{1}^{2}}\right) \delta_{1}^{2}+\frac{b_{12}^{2}(1-\psi)^{2}}{\left(1-\rho_{2}\right)^{2}}\left(l_{2}+\frac{\rho_{2}\left(1-\rho_{2}^{l_{2}}\right)\left(\rho_{2}^{l_{2}+1}-\rho_{2}-2\right)}{1-\rho_{2}^{2}}\right) \delta_{2}^{2}} .
\end{aligned}
$$

Lemma 6. Using the MMSE forecasting technique, the estimate of the standard deviation of the $l_{2}$ period forecast error of $R 2 \widehat{\xi}_{2, t}^{2}$ is a constant and can be expressed as follows:

$$
\hat{\xi}_{2, t}^{2}=\sqrt{l_{2} \sigma_{2}^{2}+l_{1} \sigma_{1}^{2}+\frac{b_{21}^{2} \psi^{2}}{\left(1-\rho_{2}\right)^{2}}\left(l_{2}+\frac{\rho_{2}\left(1-\rho_{2}^{l_{2}}\right)\left(\rho_{2}^{l_{2}+1}-\rho_{2}-2\right)}{1-\rho_{2}^{2}}\right) \delta_{2}^{2}+\frac{b_{22}^{2}(1-\psi)^{2}}{\left(1-\rho_{1}\right)^{2}}\left(l_{1}+\frac{\rho_{1}\left(1-\rho_{1}^{l_{1}}\right)\left(\rho_{1}^{l_{1}+1}-\rho_{1}-2\right)}{1-\rho_{1}^{2}}\right) \delta_{1}^{2}} .
$$


Proof. Proof

$$
\begin{aligned}
& \widehat{\xi}_{2, t}^{l_{2}}=\sqrt{\operatorname{Var}\left(\hat{d}_{2, t}^{l_{2}}-d_{2, t}^{l_{2}}\right)}=\sqrt{\operatorname{Var}\left(\sum_{i=0}^{l_{2}-1} d_{2, t}-\widehat{d}_{2, t}\right)} \\
& =\sqrt{\operatorname{Var}\left(\sum_{i=0}^{l_{2}-1}\left(-b_{21} \psi \sum_{j=0}^{i} \rho_{2}^{i-j} \eta_{2, t+j}-b_{22}(1-\psi) \sum_{j=0}^{t} \rho_{1}^{i-j} \eta_{1, t+j}+\varepsilon_{1, t+i}+\varepsilon_{2, t+i}\right)\right)} \\
& =\sqrt{\operatorname{Var}\left(\sum_{i=0}^{l_{1}-1} \varepsilon_{1, t+i}\right)+\operatorname{Var}\left(\sum_{i=0}^{l_{2}-1} \varepsilon_{2, t+i}\right)+b_{21}^{2} \psi^{2} \operatorname{Var}\left(\sum_{i=0}^{l_{2}-1} \sum_{j=0}^{i} \rho_{2}^{i-j} \eta_{2, t+j}\right)+b_{22}^{2}(1-\psi)^{2} \operatorname{Var}\left(\sum_{i=0}^{l_{1}-1} \sum_{j=0}^{i} \rho_{1}^{i-j} \eta_{1, t+j}\right)} \\
& =\sqrt{l_{1} \sigma_{1}^{2}+l_{2} \sigma_{2}^{2}+b_{21}^{2} \psi^{2} \operatorname{Var}\left(\sum_{i=0}^{l_{2}-1} \eta_{2, t+j} \sum_{j=0}^{l_{2}-1-i} \rho_{2}^{j}\right)+b_{22}^{2}(1-\psi)^{2} \operatorname{Var}\left(\sum_{i=0}^{l_{1}-1} \eta_{1, t+j} \sum_{j=0}^{l_{1}-1-i} \rho_{1}^{j}\right)} \\
& =\sqrt{l_{1} \sigma_{1}^{2}+l_{2} \sigma_{2}^{2}+\frac{b_{21}^{2} \psi^{2}}{\left(1-\rho_{2}\right)^{2}}\left(l_{2}+\frac{\rho_{2}\left(1-\rho_{2}^{l_{2}}\right)\left(\rho_{2}^{l_{2}+1}-\rho_{2}-2\right)}{1-\rho_{2}^{2}}\right) \delta_{2}^{2}+\frac{b_{22}^{2}(1-\psi)^{2}}{\left(1-\rho_{1}\right)^{2}}\left(l_{1}+\frac{\rho_{1}\left(1-\rho_{1}^{l_{1}}\right)\left(\rho_{1}^{l_{1}+1}-\rho_{1}-2\right)}{1-\rho_{1}^{2}}\right) \delta_{1}^{2}} .
\end{aligned}
$$

Theorem 6. For two parallel supply chains in which there is a competition effect on customer demand and the retailers' market share, when two retailers use the order-up-to inventory strategy and the MMSE forecasting technique, the quantitative model of the inventory bullwhip effect in two supply chains is expressed as follows:

$$
\begin{gathered}
I B E_{\text {retailer-1 }}^{\text {MMSE }}=\frac{\operatorname{Var}\left(I_{1, t}\right)}{\operatorname{Var}\left(d_{1, t}\right)}=\frac{l_{1} \sigma_{1}^{2}+l_{2} \sigma_{2}^{2}+\left(b_{11}^{2} \psi^{2}\right) /\left(1-\rho_{1}\right)^{2}\left(l_{1}+\left(\left(\rho_{1}\left(1-\rho_{1}^{l_{1}}\right)\left(\rho_{1}^{l_{1}+1}-\rho_{1}-2\right)\right) /\left(1-\rho_{1}^{2}\right)\right)\right) \delta_{1}^{2}+\left(b_{12}^{2}(1-\psi)^{2}\right) /\left(\left(1-\rho_{2}\right)^{2}\right)\left(l_{2}+\left(\rho_{2}\left(1-\rho_{2}^{l_{2}}\right)\left(\rho_{2}^{l_{2}+1}-\rho_{2}-2\right)\right) /\left(1-\rho_{2}^{2}\right)\right) \delta_{2}^{2}}{\delta_{1, d}^{2}}, \\
\operatorname{IBE}_{\text {retailer-2 }}^{\text {MMSE }}=\frac{\operatorname{Var}\left(I_{2, t}\right)}{\operatorname{Var}\left(d_{2, t}\right)}=\frac{l_{2} \sigma_{2}^{2}+l_{1} \sigma_{1}^{2}+\left(b_{21}^{2} \psi^{2}\right) /\left(1-\rho_{2}\right)^{2}\left(l_{2}+\left(\left(\rho_{2}\left(1-\rho_{2}^{l_{2}}\right)\left(\rho_{2}^{l_{2}+1}-\rho_{2}-2\right)\right) /\left(1-\rho_{2}^{2}\right)\right)\right) \delta_{2}^{2}+\left(b_{22}^{2}(1-\psi)^{2}\right) /\left(\left(1-\rho_{1}\right)^{2}\right)\left(l_{1}+\left(\rho_{1}\left(1-\rho_{1}^{l_{1}}\right)\left(\rho_{1}^{l_{1}+1}-\rho_{1}-2\right)\right) /\left(1-\rho_{1}^{2}\right)\right) \delta_{1}^{2}}{\delta_{2, d}^{2}} .
\end{gathered}
$$

\section{Simulation and Result Analysis}

6.1. Simulation Process. From the above theorems, we obtained the quantitative model of the bullwhip effect and the inventory bullwhip effect in two parallel supply chains. This can be contrasted with the work by Ma et al. [7], which compared the bullwhip effect on product orders and on inventory by employing the three forecasting techniques in a two-level supply chain. The research used the numerical method and included one manufacturer and one retailer. However, in this section, we analyze the impact of different forecasting techniques on the bullwhip effect and on the inventory bullwhip effect in two parallel supply chains using ExtendSim Software 7.1. Thus, the simulation model of the bullwhip effect is constructed on the basis of the MA, ES, and MMSE forecasting techniques in Figures 2-4, respectively. The simulation model of the inventory bullwhip effect is constructed on the basis of the MA, ES, and MMSE forecasting techniques in Figures 5-7, respectively.
We should explain the meaning of Figure 2. The selling price of the product of both retailers can be expressed as $p_{1, t}=\psi \mu_{1}+$ $\rho_{1} p_{1, t-1}+\psi \eta_{1, t}$ and $p_{2, t}=(1-\psi) \mu_{2}+\rho_{2} p_{2, t-1}+(1-\psi) \eta_{2, t}$, respectively. The demand model which $\mathrm{R} 1$ faces can be expressed as $d_{1, t}=a_{1}-b_{11} p_{1, t}+b_{12} p_{2, t}+\varepsilon_{1, t}$. The R1's order to manufacturer- 1 can be expressed as $q_{1, t}=\left(1+l_{1} / k\right) d_{1, t-1}-$ $\left(l_{1} / k\right) d_{1, t-k-1}$. Finally, the bullwhip effect can be expressed as $\mathrm{BE}_{\text {retailer-1 }}^{\mathrm{MA}}=\operatorname{Var}\left(q_{1, t}\right) / \operatorname{Var}\left(d_{1, t}\right)$. Figure 3 indicates that the selling price of the product of both retailers can be expressed as $p_{1, t}=\psi \mu_{1}+\rho_{1} p_{1, t-1}+\psi \eta_{1, t}$ and $p_{2, t}=(1-\psi) \mu_{2}+\rho_{2} p_{2, t-1}+$ $(1-\psi) \eta_{2, t}$, respectively. The demand model which R1 faces can be expressed as $d_{1, t}=a_{1}-b_{11} p_{1, t}+b_{12} p_{2, t}+\varepsilon_{1, t}$. R1's order to manufacturer- 1 can be expressed as $q_{1, t}=\left(1+\alpha l_{1}\right) d_{1, t}$ $\alpha l_{1} d t g_{1}$. The bullwhip effect can be calculated using the following equation: $\mathrm{BE}_{\text {retailer-1 }}^{\mathrm{ES}}=\operatorname{Var}\left(q_{1, t}\right) / \operatorname{Var}\left(d_{1, t}\right)$. Figure 4 illustrates that the selling price of the product of both retailers can be expressed as $\widehat{p}_{1, t+i}=E\left(p_{1, t+i} \mid p_{1, t-1}\right)=$ $\left(\left(1-\rho_{1}^{i+1}\right) / \rho_{1}\right) \mu_{1} \psi+\rho_{1}^{i+1} p_{1, t-1}$ and $\widehat{p}_{2, t+i}=E\left(p_{2, t+i} \mid p_{2, t-1}\right)=$ $\left(\left(1-\rho_{2}^{i+1}\right) / \quad \rho_{2}\right) \mu_{2}(1-\psi)+\rho_{2}^{i+1} p_{2, t-1}$, respectively. The 


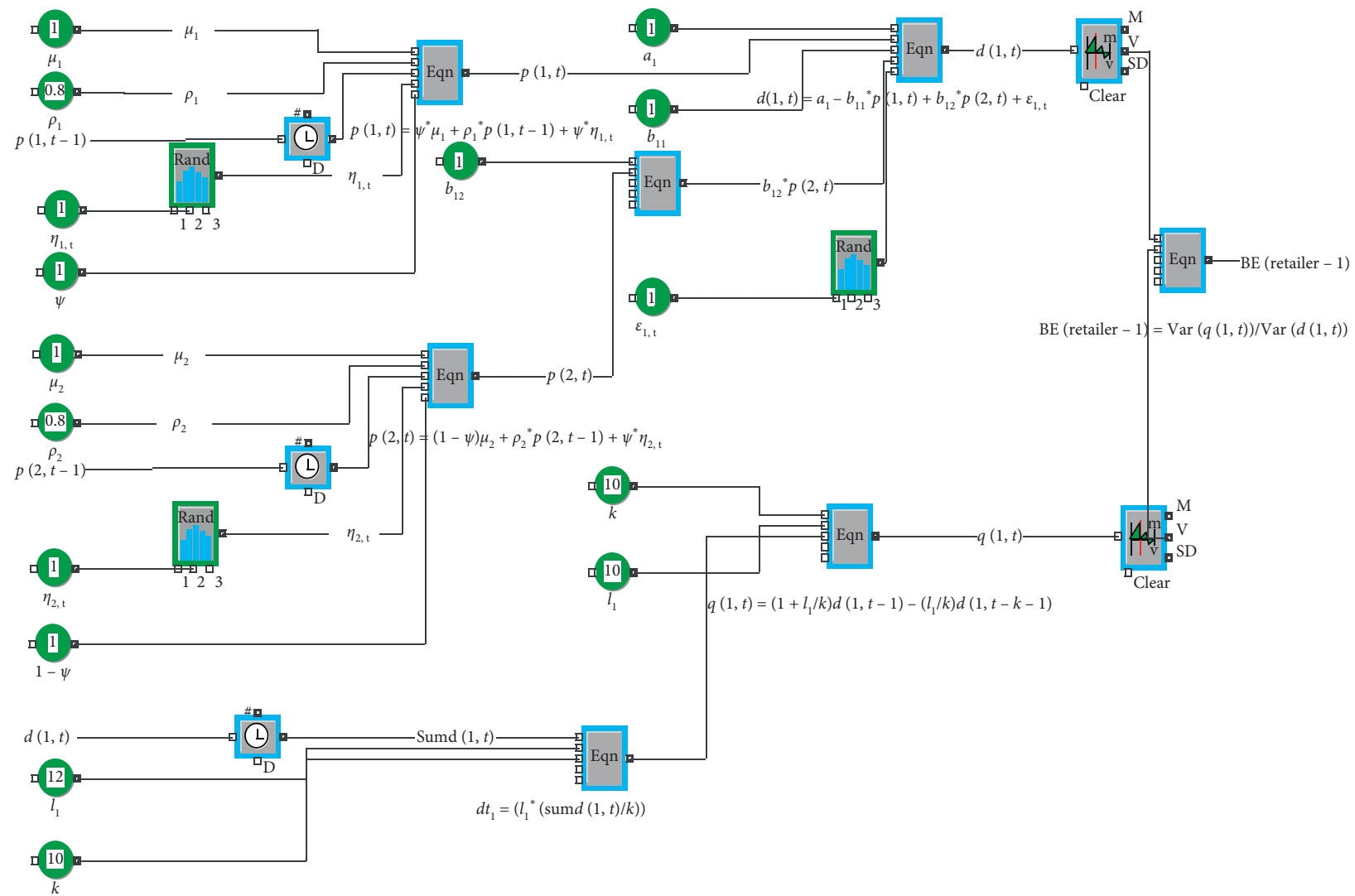

Figure 2: The simulation model of the bullwhip effect based on the MA technique.

demand model which R1 faces can be expressed as $d_{1, t+i}=$ $a_{1}-b_{11} p_{1, t+i}+b_{12} p_{2, t+i}$. R1's order to manufacturer-1 is as follows: $q_{1, t}=-b_{11} \rho_{1}\left(\rho_{1} \psi\left(1-\rho_{1}^{l_{1}}\right)\left(p_{1, t-1}-p_{1, t-2}\right)\right) /\left(1-\rho_{1}\right)+$ $b_{12} \rho_{2}\left(\rho_{2}\left(1-\rho_{2}^{l_{1}}\right)\left(p_{2, t-1}-p_{2, t-2}\right)\right) /\left(1-\rho_{2}\right)+d_{1, t-1}$. The bullwhip effect can be calculated as follows: $\mathrm{BE}_{\text {retailer-1 }}^{\mathrm{MMSE}}=$ $\operatorname{Var}\left(q_{1, t}\right) / \operatorname{Var}\left(d_{1, t}\right)$.

The demand process, the order process, and the price process shown in Figure 5 are the same as those in Figure 2. The inventory level of R1 can then be determined as follows: $I_{1, t}=d t 1-d_{1, t}$. The inventory bullwhip effect can be calculated as follows: $\operatorname{IBE}_{\text {retailer-1 }}^{\mathrm{MA}}=\operatorname{Var}\left(I_{1, t}\right) / \operatorname{Var}\left(d_{1, t}\right)$. The demand process, the order process, and the price process in Figure 6 are the same as those in Figure 3. R1's inventory level can then be calculated as follows: $I_{1, t}=d t 1-d t g 1$. The inventory bullwhip effect can be determined using the following equation: $\mathrm{IBE}_{\text {retailer-1 }}^{\mathrm{ES}}=\operatorname{Var}\left(I_{1, t}\right) / \operatorname{Var}\left(d_{1, t}\right)$. The demand process, the order process, and the price process in Figure 7 are the same as those in Figure 4. R1's inventory level can then be calculated as follows: $I_{1, t}=d t 1-d(1, t)$. The inventory bullwhip effect can be formulated using the equation: $\operatorname{IBE}_{\text {retailer-1 }}^{\text {MMSE }}=\operatorname{Var}\left(I_{1, t}\right) / \operatorname{Var}\left(d_{1, t}\right)$.

\subsection{Analysis of Results}

6.2.1. Comparing the Bullwhip Effect Using Different Forecasting Techniques. Based on the quantitative model and the simulation model of the bullwhip effect using different forecasting techniques, we found that the bullwhip effect for R1, using different forecasting techniques, depends on the self-price sensitivity coefficient, the cross-price sensitivity coefficient, the price autoregressive coefficients, the market share, and the variance. However, the bullwhip effect cannot be impacted by the potential market demand. We analyzed the impact of different forecasting techniques on the bullwhip effect for R1. The relevant parameters can be applied as follows: $\mu_{1}=1, \mu_{2}=1, \delta_{1, p}^{2}=\delta_{2, p}^{2}=1$, and $\delta_{12}^{2}=0.1$ (Tables 2 and 3 ).

The following conclusion can be drawn from Table 4 .

(1) From No. 1-3, when the price autoregressive coefficients of the two retailers are very low and have the same value, and regardless of how much longer the lead time is, or how much larger the smoothing coefficient is, or how bigger the market share is, R1's bullwhip effect is at the lowest level using the MMSE forecasting technique.

(2) From No. 4-9, when R1's self-price sensitivity coefficient and R1's cross-price sensitivity coefficient are at the low level and have the same value, other variables are equal to any values, and R1's bullwhip effect is at the lowest level using the MMSE forecasting technique.

(3) From No. 10-15, when R1's self-price sensitivity coefficient, cross-price sensitivity coefficient, lead 


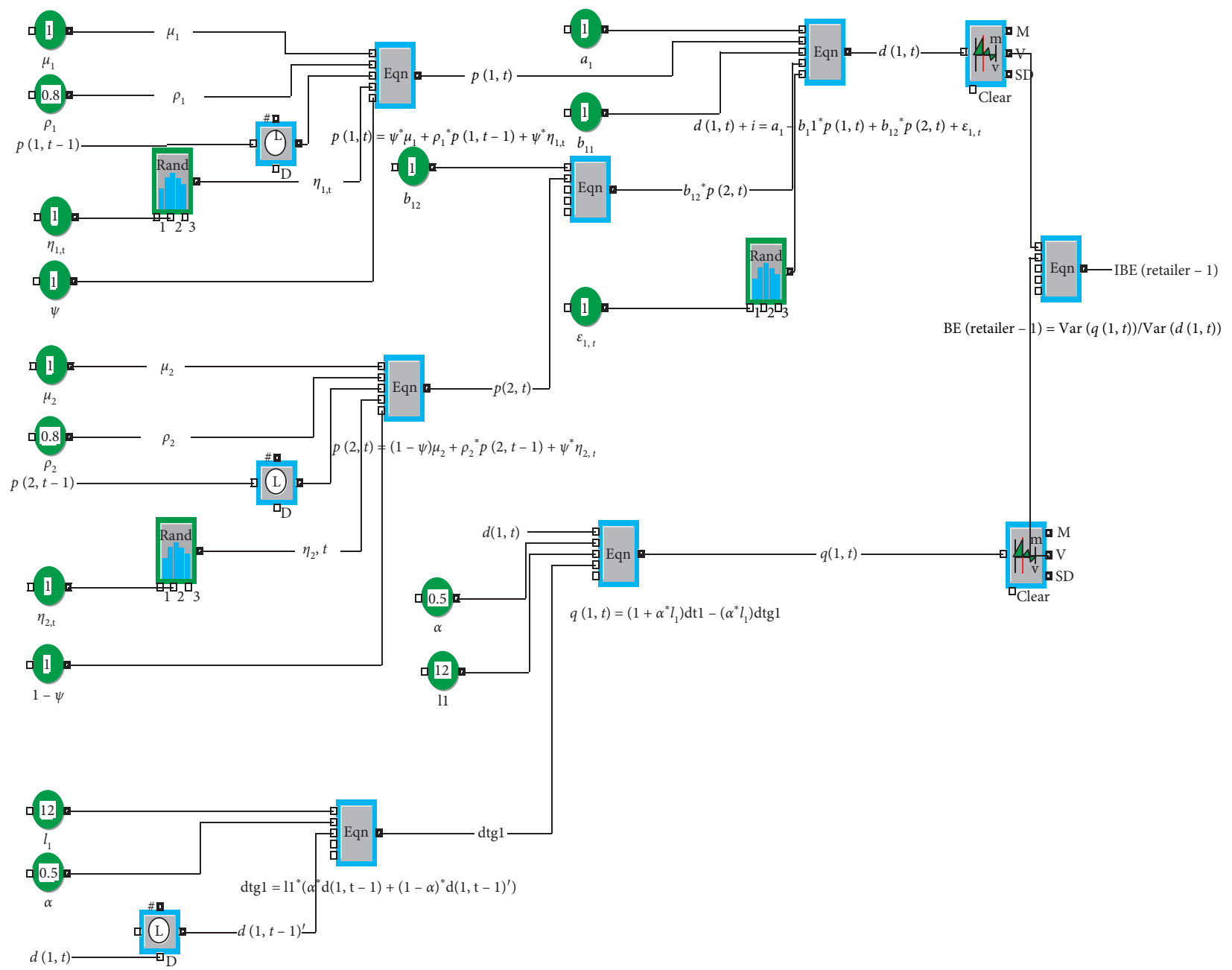

Figure 3: The simulation model of the bullwhip effect based on the ES technique.

time, market share, smoothing coefficient, and price autoregressive coefficient are from low to high, R1's bullwhip effect is at the highest level using the MA forecasting technique.

(4) From No. 16-19, when R1's self-price sensitivity coefficient, cross-price sensitivity coefficient, lead time, market share, smoothing coefficient, and price autoregressive coefficient are all at the low level, R1's bullwhip effect is at the lowest level using the MMSE forecasting technique.

We converted the data into figures in order to analyze the impact of the relevant factors on R1's bullwhip effect. Moreover, we can obtain some important managerial insights from figures. Figure 8 shows the impact of $b_{11}$ and $b_{12}$ on the bullwhip effect using different forecasting techniques. Figure 9 depicts the impact of $\rho_{1}$ and $\rho_{2}$ on the bullwhip effect. Figure 10 depicts the impact of $\psi$ on the bullwhip effect.

By comparing the bullwhip effect using different forecasting techniques, as shown in Figures 8-10, we can conclude the following: (1) as the MMSE forecasting techniques can reduce the lead-time demand forecast error to the largest extent, the bullwhip effect can reach the lowest level using the MMSE techniques; (2) R1 can reduce the bullwhip effect using the MA techniques when the self-price sensitivity coefficient $b_{11}$ is lower than 5.5, the price autoregressive coefficient $\rho_{1}$ is lower than 0.45 , and the market share $\psi$ is lower than 0.4 . On the contrary, $\mathrm{R} 1$ can reduce the bullwhip effect using the ES techniques. Moreover, $\Delta \mathrm{BE}_{\text {retailer-1 }}=$ $\mathrm{BE}_{\text {retailer-1 }}^{\mathrm{MA}}-\mathrm{BE}_{\text {retailer-1}}^{\mathrm{ES}}$, when $\sigma_{1}^{2}=\delta_{1}^{2}, \sigma_{2}^{2}=\delta_{2}^{2}$, and we can determine the following:

$$
\begin{aligned}
\Delta \mathrm{BE}_{\text {retailer }}= & b_{11} b_{12} \frac{(1-\psi)}{1-\rho_{1} \rho_{2}} \frac{\rho_{1}}{1-\rho_{1}}\left(l_{1}-\frac{1-\rho_{1}^{l_{1}}}{1-\rho_{1}}\right) \\
& +b_{11}^{2} \rho_{1} \frac{\left(l_{1}-\left(1-\rho_{1}^{l_{1}}\right)\right)}{\left(1-\rho_{1}\right)^{2}}+\rho_{1} \frac{l_{1}^{2}}{\left(1-\rho_{1}\right)} \\
& +\frac{l_{2}\left(1-\rho_{2}^{l_{2}}\right)}{\left(1-\rho_{2}\right)}<\frac{1}{2 \psi}\left(\left(\frac{l_{1}}{k}\right)^{2}+\left(l_{1}^{2} \frac{\alpha}{2-\alpha}\right)\right) .
\end{aligned}
$$




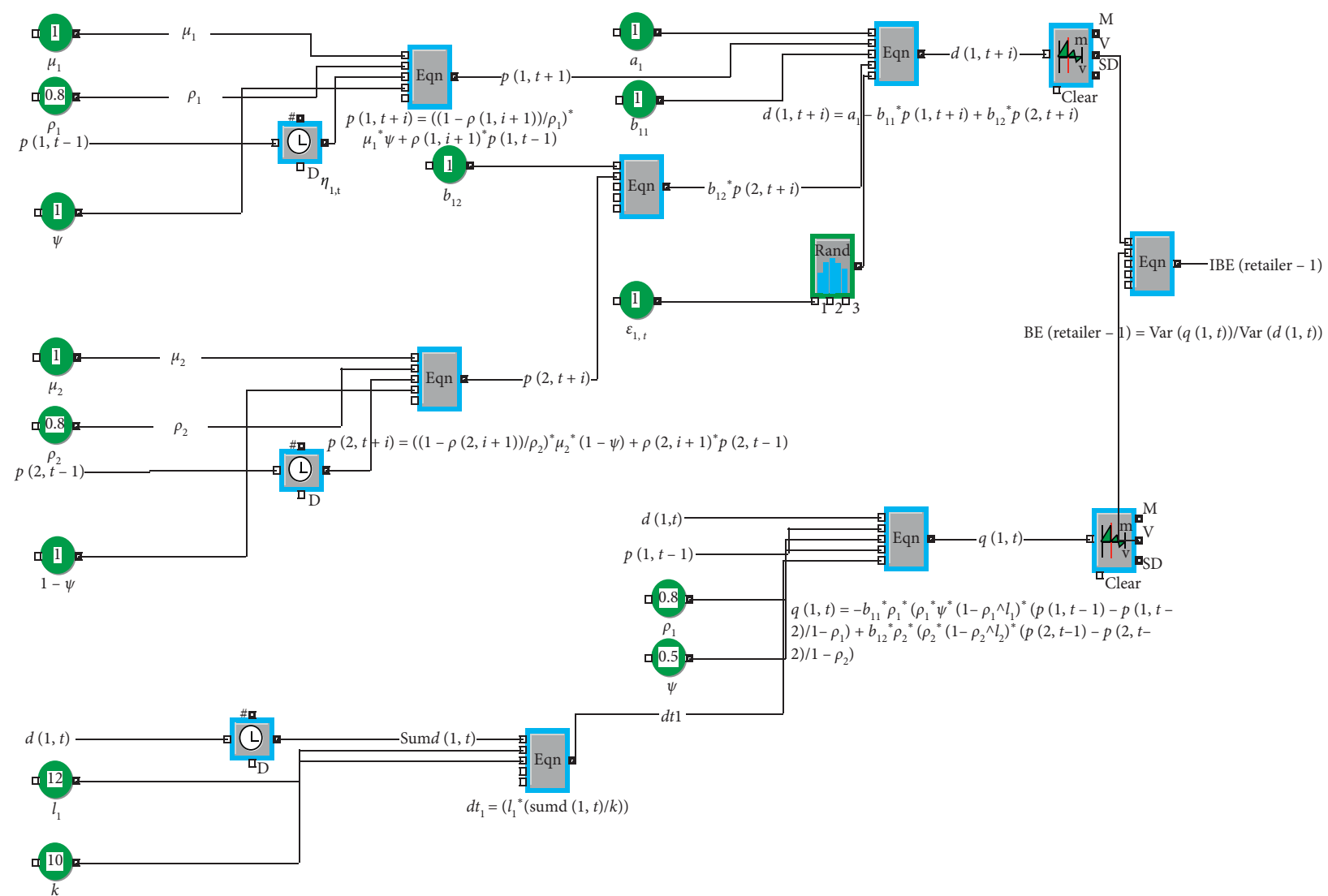

FIgURE 4: The simulation model of the bullwhip effect based on the MMSE technique.

Proposition 1. When the self-price sensitivity coefficient $b_{11}$, the cross-price sensitivity coefficient $b_{12}$, the price autoregressive coefficients $\rho_{1}$ and $\rho_{2}$, and the market share $\psi$ satisfy the following condition, $R 1$ can control the bullwhip effect using the MA technique. Otherwise, R1 should select the ES technique.

$$
b_{11} b_{12} \frac{(1-\psi)}{1-\rho_{1} \rho_{2}} \frac{\rho_{1}}{1-\rho_{1}}\left(l_{1}-\frac{1-\rho_{1}^{l_{1}}}{1-\rho_{1}}\right)+b_{11}^{2} \rho_{1} \frac{\left(l_{1}-\left(1-\rho_{1}^{l_{1}}\right)\right)}{\left(1-\rho_{1}\right)^{2}}+\rho_{1} \frac{l_{1}^{2}}{\left(1-\rho_{1}\right)}+\frac{l_{2}\left(1-\rho_{2}^{l_{2}}\right)}{\left(1-\rho_{2}\right)}<\frac{1}{2 \psi}\left(\left(\frac{l_{1}}{k}\right)^{2}+\left(l_{1}^{2} \frac{\alpha}{2-\alpha}\right)\right)
$$

It is evident that the potential market demand $a_{1}$ has no impact on R1's bullwhip effect. Thus, the potential market demand cannot influence R1's choice of the forecasting technique. On the other hand, when the self-price sensitivity coefficient $b_{11}$, the cross-price sensitivity coefficient $b_{12}$, the price autoregressive coefficients $\rho_{1}$ and $\rho_{2}$, and the market share $\psi$ satisfy Proposition 1, R1 can control the bullwhip effect using the MA technique. Otherwise, R1 should select the ES technique.

6.2.2. Comparing the Inventory Bullwhip Effect Using Different Forecasting Techniques. Based on the quantitative model and the simulation model of the inventory bullwhip effect using different forecasting techniques, we found that R1's inventory bullwhip effect using different forecasting techniques depends on the self-price sensitivity coefficient, the cross-price sensitivity coefficient, the price autoregressive coefficients, the market share, and the variance. However, it cannot be impacted by the potential market demand. We analyzed the impact of different forecasting techniques on R1's inventory bullwhip effect. The relevant parameters can be applied as follows: $\mu_{1}=1, \mu_{2}=1, \delta_{1, p}^{2}=\delta_{2, p}^{2}=1$, and $\delta_{12}^{2}=0.1$ (Tables 5 and 6). The following conclusion can be drawn from Table 7.

(1) In No. 1-3, when the value of the two retailers' price autoregressive coefficient is very low and at the same level, regardless of the length of the lead time, or how much larger the smoothing coefficient is, or how much larger the market share is, R1's inventory bullwhip effect is at the lowest level using the MMSE forecasting technique.

(2) In No. 4-9, when R1's self-price sensitivity coefficient and R1's cross-price sensitivity coefficient are at 


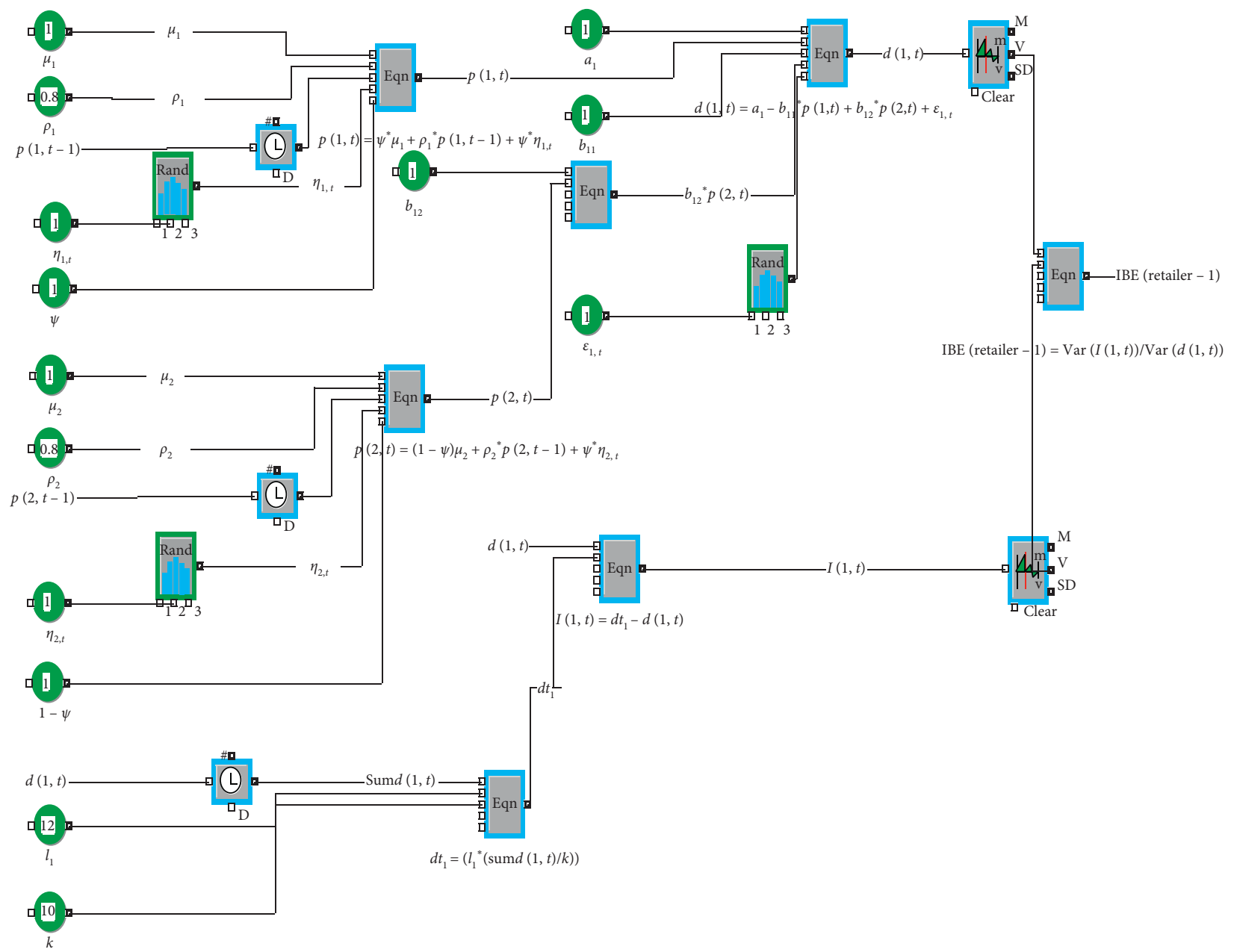

Figure 5: The simulation model of the inventory bullwhip effect based on the MA technique.

the low level and at the same level, regardless of the length of the lead time, or how much larger the smoothing coefficient is, or how much larger the market share is, R1's inventory bullwhip effect is at the lowest level using the MMSE forecasting technique.

(3) In No. 16-18, when the value of the two retailers' price autoregressive coefficient is very low and at a different level, and when the smoothing coefficient is very low and the lead time is very low, R1's bullwhip effect is at the lowest level using the MMSE forecasting technique. Moreover, R1's inventory bullwhip effect is at the highest level using the ES forecasting technique.

We converted the data into figures in order to analyze the impact of the relevant factors on R1's bullwhip effect. Moreover, we can gain some important managerial insights from the figures. Figure 11 depicts the impact of $b_{11}$ and $b_{12}$ on the inventory bullwhip effect. Figure 12 depicts the impact of $\rho_{1}$ and $\rho_{2}$ on the inventory bullwhip effect. Figure 13 depicts the impact of $\psi$ on the inventory bullwhip effect.
By comparing the inventory bullwhip effect using different forecasting techniques, as shown in Figures 11-13, it is evident that the inventory bullwhip effect can reach the highest level using the ES technique (Chen et al. and Zhang et al. reached the same conclusions). Moreover, R1 can control the impact of the inventory bullwhip effect using the MA technique or the MMSE technique. We can then analyze which forecasting technique R1 should select under different conditions. Figure 11 shows that the inventory bullwhip effect can achieve the lowest level under the MMSE technique when the value of the self-price sensitivity coefficient $b_{11}$ is lower than 7 . Otherwise, the inventory bullwhip effect can reach the lowest level using the MA technique when $b_{11}$ is higher than 7. Figure 12 shows that the inventory bullwhip effect can reach the lowest level using the MMSE technique when the price autoregressive coefficient $\rho_{1}<0.8$. Otherwise, the inventory bullwhip effect can reach the lowest level using the MA technique when the price autoregressive coefficient $\rho_{1}>0.8$. Figure 13 indicates that the inventory bullwhip effect can achieve the lowest level using the MA technique when the value of the market share $\psi$ is lower than 0.6. 


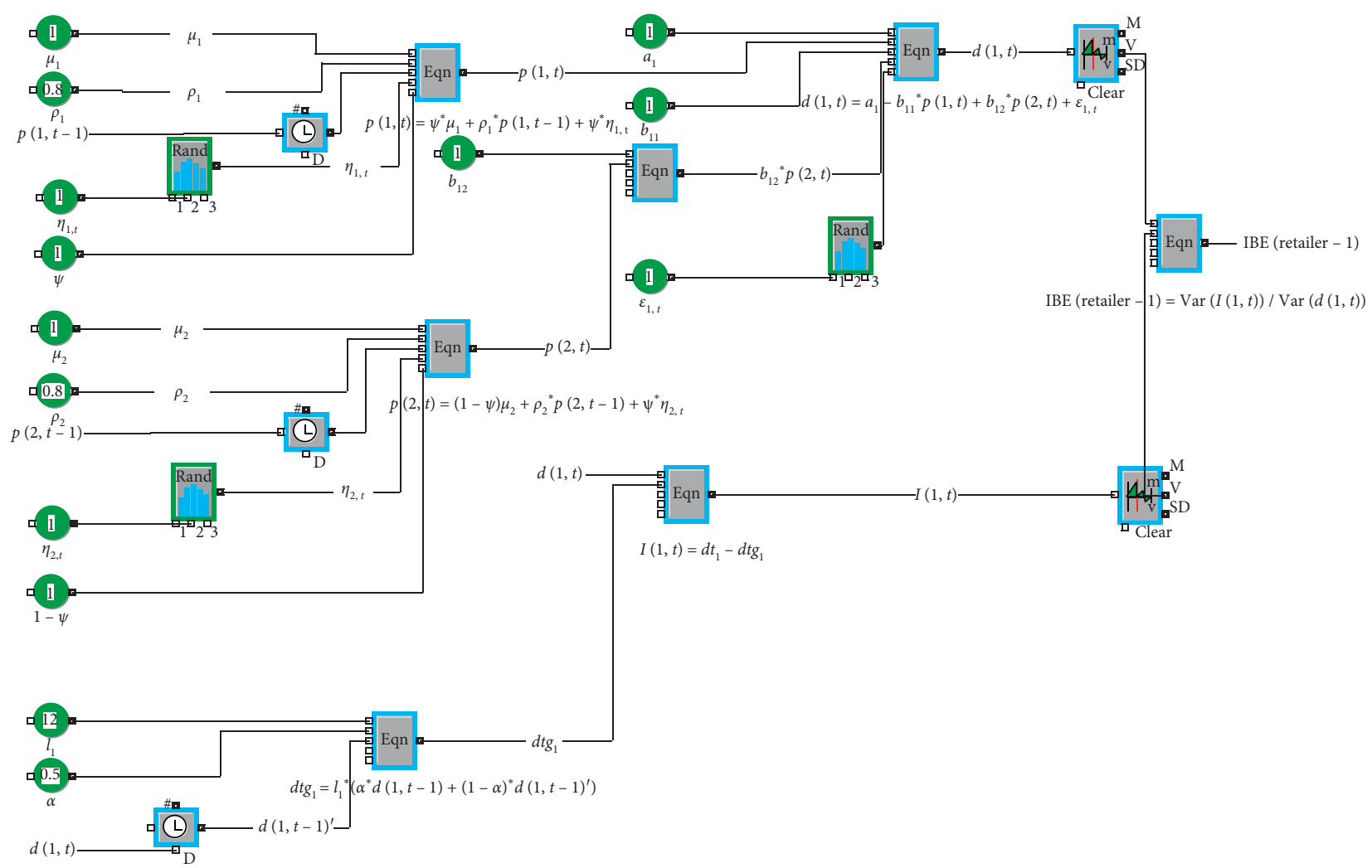

Figure 6: The simulation model of the inventory bullwhip effect based on the ES technique.

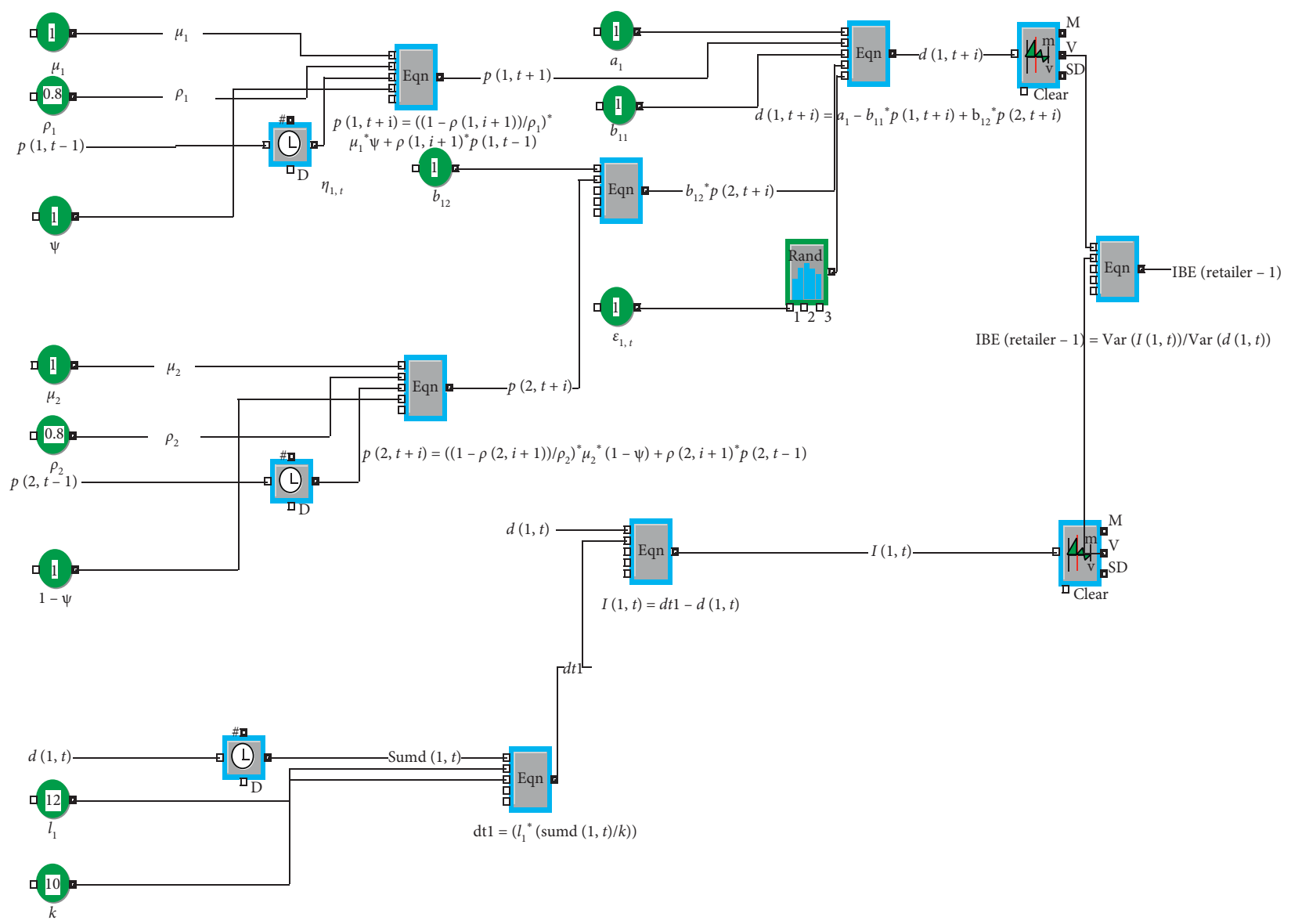

FIGURE 7: The simulation model of the inventory bullwhip effect based on the MMSE technique. 
TABLE 2: The value range of the relevant factors.

\begin{tabular}{lllcccccc}
\hline Factors & $l_{1}$ & $l_{2}$ & $k$ & $b_{11}$ & $b_{12}$ & $\psi$ & $\rho_{1}$ & $\rho_{2}$ \\
\hline Level 1 & 2 & 2 & 4 & 5 & 5 & 0.3 & 0.3 & 0.3 \\
Level 2 & 4 & 4 & 8 & 10 & 10 & 0.6 & 0.6 & 0.6 \\
Level 3 & 6 & 6 & 12 & 15 & 15 & 0.9 & 0.9 & 0.9 \\
Level 4 & 2 & 2 & 4 & 5 & 5 & 0.3 & 0.3 & 0.6 \\
Level 5 & 2 & 2 & 4 & 5 & 5 & 0.3 & 0.3 & 0.9 \\
Level 6 & 2 & 2 & 4 & 5 & 5 & 0.3 & 0.3 & 0.3 \\
Level 7 & 2 & 2 & 4 & 5 & 5 & 0.3 & 0.3 & 0.3 \\
\hline
\end{tabular}

TABle 3: The simulation number.

\begin{tabular}{lccccccccc}
\hline No. & $l_{1}$ & $l_{2}$ & $k$ & $b_{11}$ & $b_{12}$ & $\psi$ & $\rho_{1}$ & $\rho_{2}$ \\
\hline 1 & $1(2)$ & $1(2)$ & $1(4)$ & $1(5)$ & $1(5)$ & $1(0.3)$ & $1(0.3)$ & $1(0.3)$ & $1(0.3)$ \\
2 & $2(4)$ & $2(4)$ & $2(8)$ & $2(10)$ & $2(10)$ & $2(0.6)$ & $2(0.3)$ & $2(0.3)$ & $2(0.6)$ \\
3 & $3(6)$ & $3(6)$ & $3(12)$ & $3(15)$ & $3(15)$ & $3(0.9)$ & $3(0.3)$ & $3(0.3)$ & $3(0.9)$ \\
4 & $1(2)$ & $1(2)$ & $1(4)$ & $1(5)$ & $1(5)$ & $1(0.3)$ & $1(0.6)$ & $1(0.6)$ & $1(0.3)$ \\
5 & $2(4)$ & $2(4)$ & $2(8)$ & $2(5)$ & $2(5)$ & $2(0.6)$ & $2(0.6)$ & $2(0.6)$ & $2(0.6)$ \\
6 & $3(6)$ & $3(6)$ & $3(12)$ & $3(5)$ & $3(5)$ & $3(0.9)$ & $3(0.6)$ & $3(0.6)$ & $3(0.9)$ \\
7 & $1(2)$ & $1(2)$ & $1(4)$ & $1(5)$ & $1(5)$ & $1(0.3)$ & $1(0.9)$ & $1(0.9)$ & $1(0.3)$ \\
8 & $2(4)$ & $2(4)$ & $2(8)$ & $2(5)$ & $2(5)$ & $2(0.6)$ & $2(0.9)$ & $2(0.9)$ & $2(0.6)$ \\
9 & $3(6)$ & $3(6)$ & $3(12)$ & $3(5)$ & $3(5)$ & $3(0.9)$ & $3(0.9)$ & $3(0.9)$ & $3(0.9)$ \\
10 & $1(2)$ & $1(2)$ & $1(4)$ & $1(5)$ & $1(5)$ & $1(0.3)$ & $1(0.3)$ & $1(0.3)$ & $1(0.3)$ \\
11 & $1(2)$ & $1(2)$ & $1(4)$ & $1(5)$ & $1(5)$ & $1(0.3)$ & $1(0.3)$ & $1(0.3)$ & $1(0.3)$ \\
12 & $2(4)$ & $2(4)$ & $2(8)$ & $2(10)$ & $2(10)$ & $2(0.6)$ & $2(0.6)$ & $2(0.6)$ & $2(0.6)$ \\
13 & $2(4)$ & $2(4)$ & $2(8)$ & $2(10)$ & $2(10)$ & $2(0.6)$ & $2(0.6)$ & $2(0.6)$ & $2(0.6)$ \\
14 & $3(6)$ & $3(6)$ & $3(12)$ & $3(15)$ & $3(15)$ & $3(0.9)$ & $3(0.9)$ & $3(0.9)$ & $3(0.9)$ \\
15 & $3(6)$ & $3(6)$ & $3(12)$ & $3(15)$ & $3(15)$ & $3(0.9)$ & $3(0.9)$ & $3(0.9)$ & $3(0.9)$ \\
16 & $4(2)$ & $4(2)$ & $4(4)$ & $4(5)$ & $4(5)$ & $4(0.3)$ & $4(0.3)$ & $4(0.6)$ & $4(0.3)$ \\
17 & $5(2)$ & $5(2)$ & $5(4)$ & $5(5)$ & $5(5)$ & $5(0.3)$ & $5(0.3)$ & $5(0.6)$ & $5(0.3)$ \\
18 & $6(2)$ & $6(2)$ & $6(4)$ & $6(5)$ & $6(5)$ & $6(0.3)$ & $6(0.3)$ & $6(0.6)$ & $6(0.3)$ \\
19 & $7(2)$ & $7(2)$ & $7(4)$ & $7(5)$ & $7(5)$ & $7(0.3)$ & $7(0.3)$ & $7(0.6)$ \\
\hline
\end{tabular}

TABLE 4: The simulation result.

\begin{tabular}{lccc}
\hline No. & $\mathrm{BE}_{\text {retailer-1 }}^{\mathrm{MA}}$ & $\mathrm{BE}_{\text {retailer-1 }}^{\mathrm{ES}}$ & $\mathrm{BE}_{\text {retailer-1 }}^{\mathrm{MMSE}}$ \\
\hline 1 & 3.4 & 5.1 & 2.1 \\
2 & 3.41 & 5.2 & 2.3 \\
3 & 3.43 & 5.25 & 2.6 \\
4 & 3.51 & 5.41 & 2.7 \\
5 & 3.53 & 5.81 & 2.8 \\
6 & 3.56 & 5.9 & 2.9 \\
7 & 3.8 & 6.02 & 3.0 \\
8 & 3.9 & 6.05 & 3.05 \\
9 & 4.0 & 6.1 & 3.1 \\
10 & 14.1 & 6.21 & 3.16 \\
11 & 14.15 & 6.3 & 3.22 \\
12 & 15.7 & 6.4 & 3.27 \\
13 & 16.1 & 6.5 & 3.37 \\
14 & 17.2 & 6.6 & 3.4 \\
15 & 17.5 & 6.9 & 3.6 \\
16 & 3.68 & 7.01 & 3.41 \\
18 & 3.72 & 7.13 & 3.3 \\
\hline
\end{tabular}




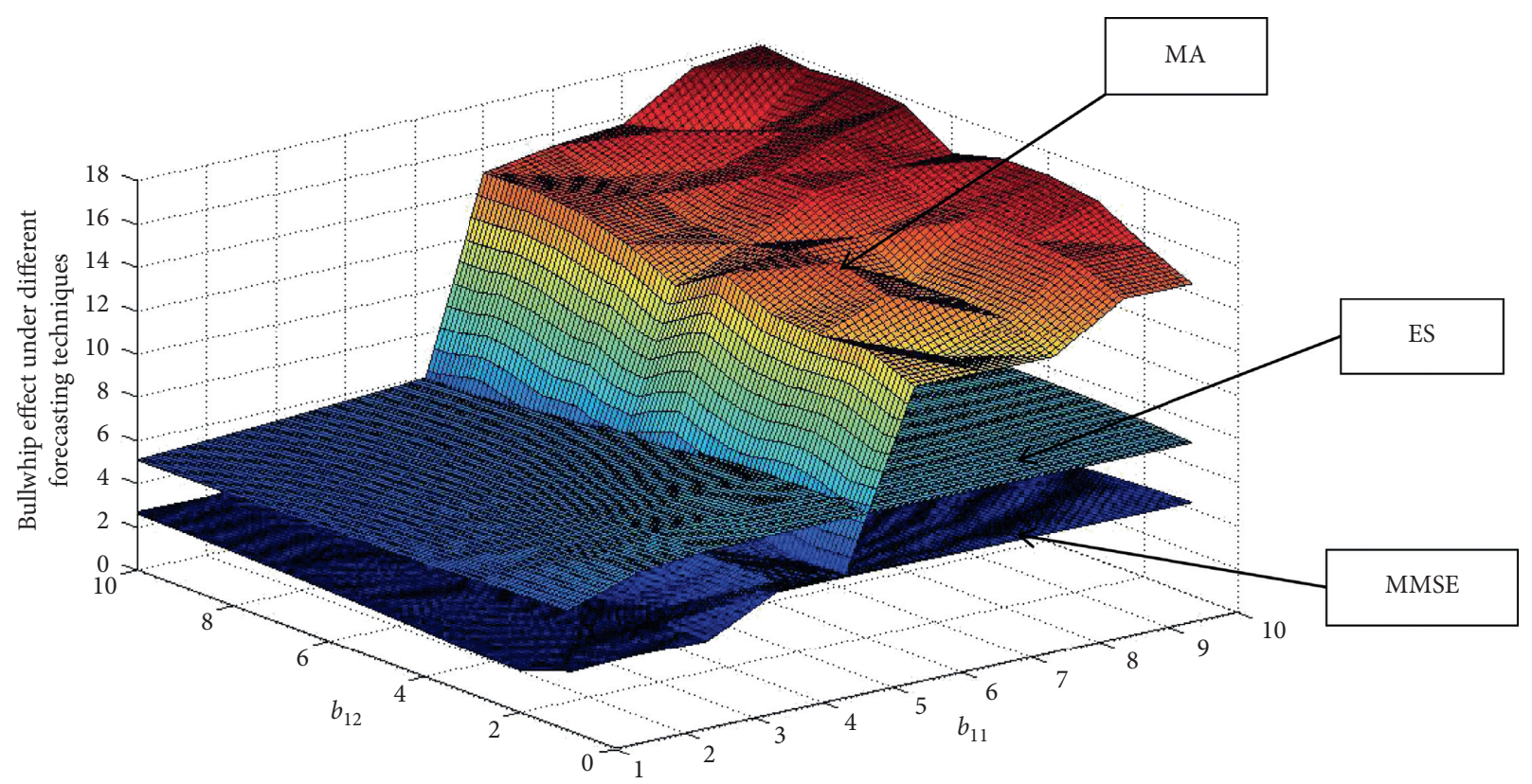

FIgURE 8: The impact of $b_{11}$ and $b_{12}$ on the bullwhip effect using different forecasting techniques.

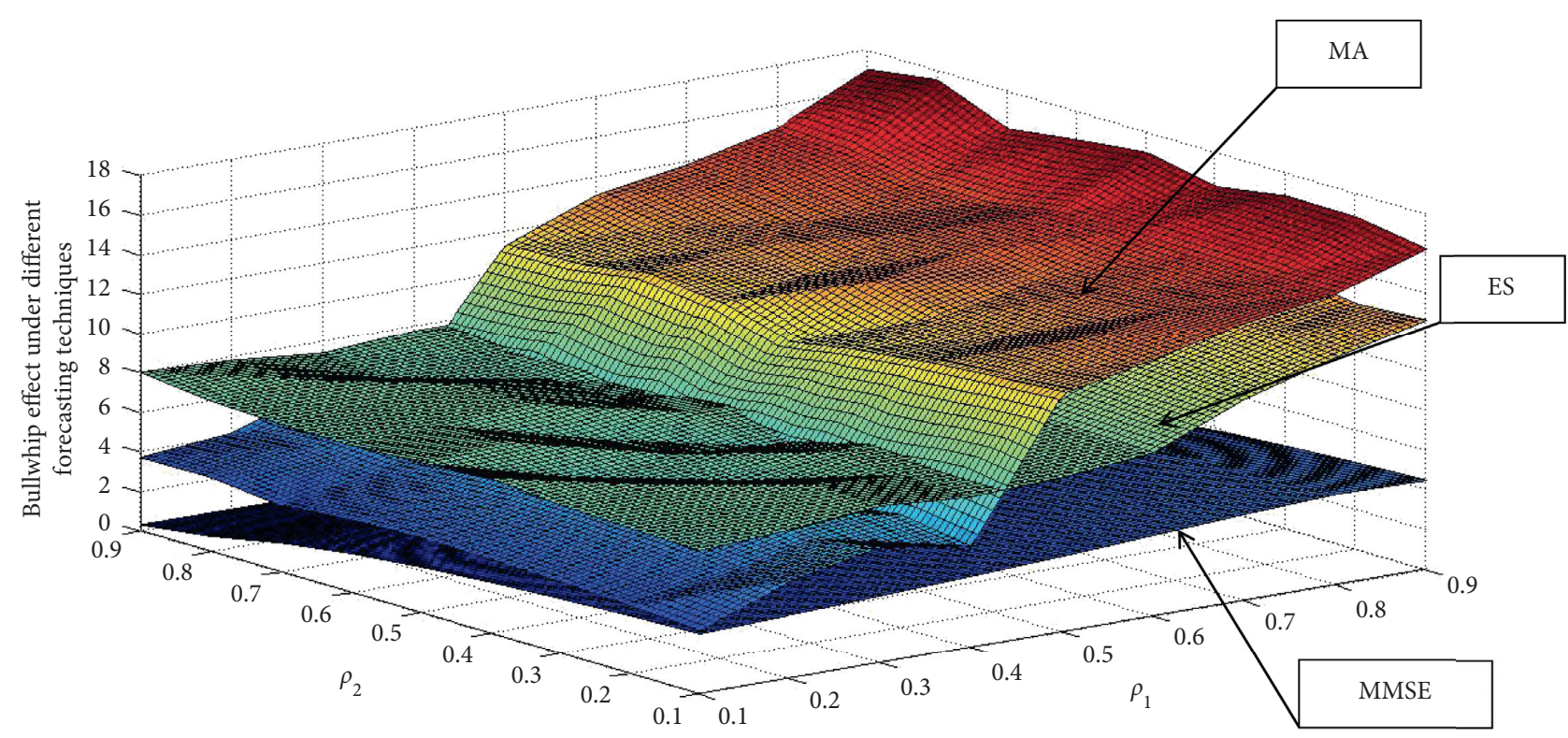

Figure 9: The impact of $\rho_{1}$ and $\rho_{2}$ on the bullwhip effect using different forecasting techniques.

Otherwise, the inventory bullwhip effect can achieve the lowest level using the MMSE technique when $\psi$ is higher than 0.6. Moreover, $\triangle \mathrm{IBE}_{\text {retailer-1 }}=\mathrm{IBE}_{\text {retailer-1 }}^{\mathrm{MMSE}}-\mathrm{IBE}_{\text {retailer-1 }}^{\mathrm{MA}}$, when $\sigma_{1}^{2}=\delta_{1}^{2}$ and $\sigma_{2}^{2}=\delta_{2}^{2}$, and we can get

$$
\begin{aligned}
\Delta \mathrm{IBE}= & -2 b_{11} b_{12}\left(\left(\rho_{1} \frac{\rho_{2}\left(1-\rho_{1}\right)}{1-\rho_{1} \rho_{2}} \frac{\rho_{2}\left(1-\rho_{2}^{l_{1}}\right)}{1-\rho_{2}^{2}}\right)+\left(\rho_{2} \frac{\rho_{1}\left(1-\rho_{2}\right)}{1-\rho_{1} \rho_{2}} \frac{\rho_{1}\left(1-\rho_{1}^{l_{1}}\right)}{1-\rho_{1}^{2}}\right)\right) \\
& +\left[2\left(\frac{\rho_{1}}{1-\rho_{1}}\right)^{2}+2 \frac{\rho_{1}}{1-\rho_{1}}\right]-2\left(1+\frac{\rho_{1}}{1-\rho_{1}}\right)\left(\frac{\rho_{1}}{1-\rho_{1}}\right)\left\{b_{11} b_{12}\left(\rho_{1}^{k}+\rho_{2}^{k}\right) \frac{\psi(1-\psi)}{1-\rho_{1} \rho_{2}}\right\} .
\end{aligned}
$$




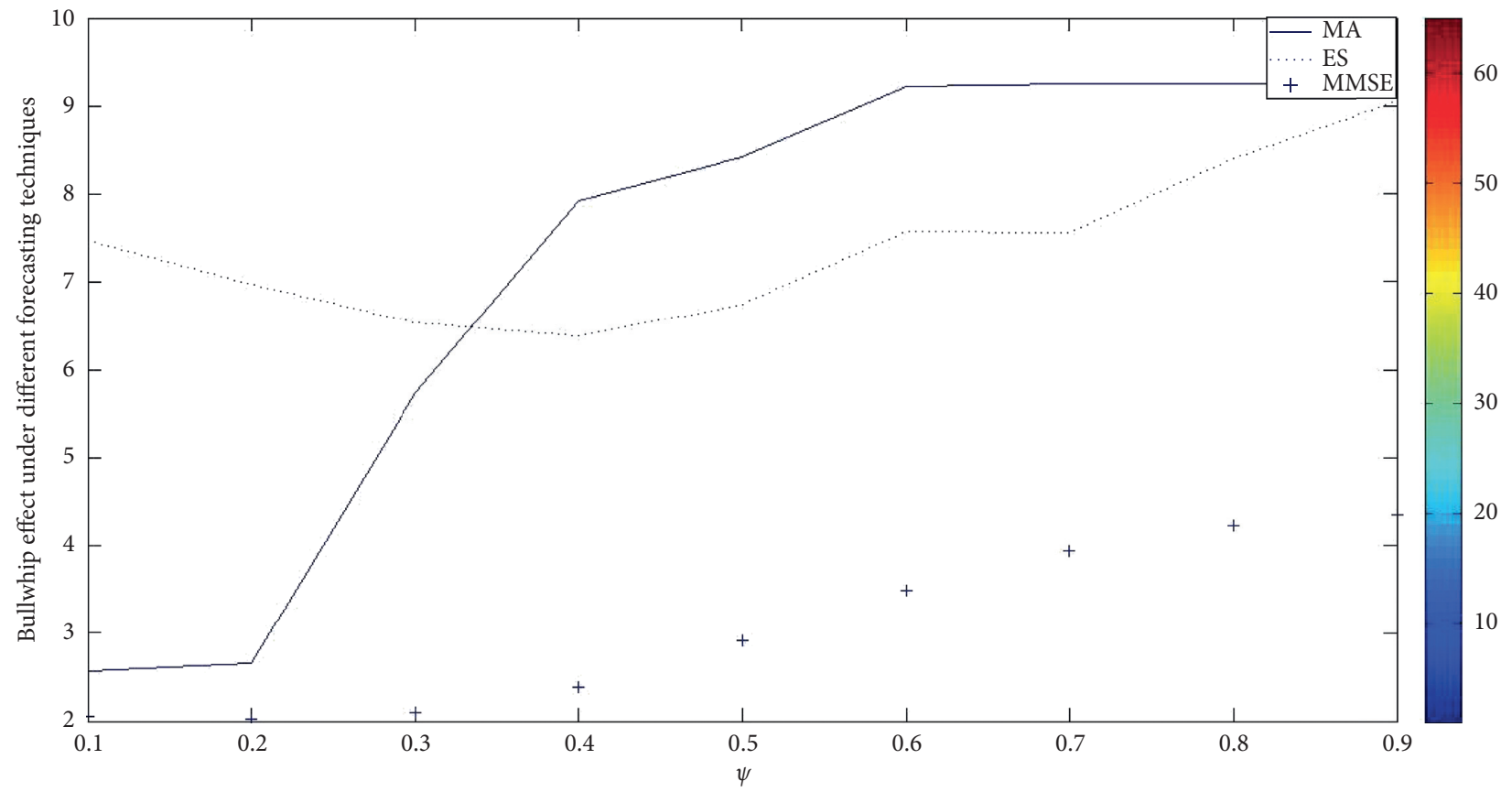

FIgURE 10: The impact of $\psi$ on the bullwhip effect using different forecasting techniques.

TABLE 5: The value range of the relevant factors.

\begin{tabular}{lccccccccc}
\hline Factors & $l_{1}$ & $l_{2}$ & $k$ & $b_{11}$ & $b_{12}$ & $\psi$ & $\rho_{1}$ & $\rho_{2}$ \\
\hline Level 1 & 2 & 2 & 4 & 5 & 5 & 0.3 & 0.3 & 0.3 \\
Level 2 & 4 & 4 & 8 & 10 & 10 & 0.6 & 0.6 & 0.6 & 0.3 \\
Level 3 & 6 & 6 & 12 & 15 & 15 & 0.9 & 0.9 & 0.9 \\
Level 4 & 2 & 2 & 4 & 5 & 5 & 0.3 & 0.3 & 0.6 & 0.9 \\
Level 5 & 2 & 2 & 4 & 5 & 5 & 0.3 & 0.3 & 0.6 \\
Level 6 & 2 & 2 & 4 & 5 & 5 & 0.3 & 0.3 & 0.3 \\
Level 7 & 2 & 2 & 4 & 5 & 5 & 0.3 & 0.3 & 0.3 \\
\hline
\end{tabular}

TABLE 6: The simulation number.

\begin{tabular}{lccccccccc}
\hline No. & $l_{1}$ & $l_{2}$ & $k$ & $b_{11}$ & $b_{12}$ & $\psi$ & $\rho_{1}$ & $\rho_{2}$ \\
\hline 1 & $1(2)$ & $1(2)$ & $1(4)$ & $1(5)$ & $1(5)$ & $1(0.3)$ & $1(0.3)$ & $1(0.3)$ & $1(0.3)$ \\
2 & $2(4)$ & $2(4)$ & $2(8)$ & $2(10)$ & $2(10)$ & $2(0.6)$ & $2(0.3)$ & $2(0.3)$ & $2(0.6)$ \\
3 & $3(6)$ & $3(6)$ & $3(12)$ & $3(15)$ & $3(15)$ & $3(0.9)$ & $3(0.3)$ & $3(0.3)$ & $3(0.9)$ \\
4 & $1(2)$ & $1(2)$ & $1(4)$ & $1(5)$ & $1(5)$ & $1(0.3)$ & $1(0.6)$ & $1(0.6)$ & $1(0.3)$ \\
5 & $2(4)$ & $2(4)$ & $2(8)$ & $2(5)$ & $2(5)$ & $2(0.6)$ & $2(0.6)$ & $2(0.6)$ & $2(0.6)$ \\
6 & $3(6)$ & $3(6)$ & $3(12)$ & $3(5)$ & $3(5)$ & $3(0.9)$ & $3(0.6)$ & $3(0.6)$ & $3(0.9)$ \\
7 & $1(2)$ & $1(2)$ & $1(4)$ & $1(5)$ & $1(5)$ & $1(0.3)$ & $1(0.9)$ & $1(0.9)$ & $1(0.3)$ \\
8 & $2(4)$ & $2(4)$ & $2(8)$ & $2(5)$ & $2(5)$ & $2(0.6)$ & $2(0.9)$ & $2(0.9)$ & $2(0.6)$ \\
9 & $3(6)$ & $3(6)$ & $3(12)$ & $3(5)$ & $3(5)$ & $3(0.9)$ & $3(0.9)$ & $3(0.9)$ & $3(0.9)$ \\
10 & $1(2)$ & $1(2)$ & $1(4)$ & $1(5)$ & $1(5)$ & $1(0.3)$ & $1(0.3)$ & $1(0.3)$ & $1(0.3)$ \\
11 & $1(2)$ & $1(2)$ & $1(4)$ & $1(5)$ & $1(5)$ & $1(0.3)$ & $1(0.3)$ & $1(0.3)$ & $1(0.3)$ \\
12 & $2(4)$ & $2(4)$ & $2(8)$ & $2(10)$ & $2(10)$ & $2(0.6)$ & $2(0.6)$ & $2(0.6)$ & $2(0.6)$ \\
13 & $2(4)$ & $2(4)$ & $2(8)$ & $2(10)$ & $2(10)$ & $2(0.6)$ & $2(0.6)$ & $2(0.6)$ & $2(0.6)$ \\
14 & $3(6)$ & $3(6)$ & $3(12)$ & $3(15)$ & $3(15)$ & $3(0.9)$ & $3(0.9)$ & $3(0.9)$ & $3(0.9)$ \\
15 & $3(6)$ & $3(6)$ & $3(12)$ & $3(15)$ & $3(15)$ & $3(0.9)$ & $3(0.9)$ & $3(0.9)$ & $3(0.9)$ \\
16 & $4(2)$ & $4(2)$ & $4(4)$ & $4(5)$ & $4(5)$ & $4(0.3)$ & $4(0.3)$ & $4(0.6)$ & $4(0.3)$ \\
17 & $5(2)$ & $5(2)$ & $5(4)$ & $5(5)$ & $5(5)$ & $5(0.3)$ & $5(0.3)$ & $5(0.6)$ & $5(0.3)$ \\
18 & $6(2)$ & $6(2)$ & $6(4)$ & $6(5)$ & $6(5)$ & $6(0.3)$ & $6(0.3)$ & $6(0.6)$ & $6(0.3)$ \\
19 & $7(2)$ & $7(2)$ & $7(4)$ & $7(5)$ & $7(5)$ & $7(0.3)$ & $7(0.3)$ & $7(0.6)$ & $7(0.6)$ \\
\hline
\end{tabular}


TABLE 7: The simulation result.

\begin{tabular}{lccc}
\hline No. & $\mathrm{IBE}_{\text {retailer-1 }}^{\mathrm{MA}}$ & $\mathrm{IBE}_{\text {retailer-1 }}^{\mathrm{ES}}$ & $\mathrm{IBE}_{\text {retailer-1 }}^{\mathrm{MMSE}}$ \\
\hline 1 & 89.6 & 130.3 & 21.9 \\
2 & 75.5 & 130.6 & 21.7 \\
3 & 74.3 & 130.8 & 21.6 \\
4 & 74.1 & 131.4 & 21.4 \\
5 & 73.8 & 131.8 & 21.1 \\
6 & 73.3 & 131.9 & 20.9 \\
7 & 73.2 & 132.2 & 20.7 \\
8 & 72.8 & 132.4 & 20.5 \\
9 & 72.6 & 132.6 & 20.2 \\
10 & 72.4 & 132.8 & 20.1 \\
11 & 72.1 & 132.9 & 1 \\
12 & 71.9 & 133.3 & 19.9 \\
13 & 71.6 & 133.6 & 19.8 \\
14 & 71.2 & 133.8 & 19.4 \\
15 & 70.8 & 133.9 & 18.9 \\
16 & 70.5 & 134.2 & 18.7 \\
17 & 69.9 & 134.6 & 18.5 \\
19 & 68.6 & 134.8 & 18.5 \\
\hline
\end{tabular}

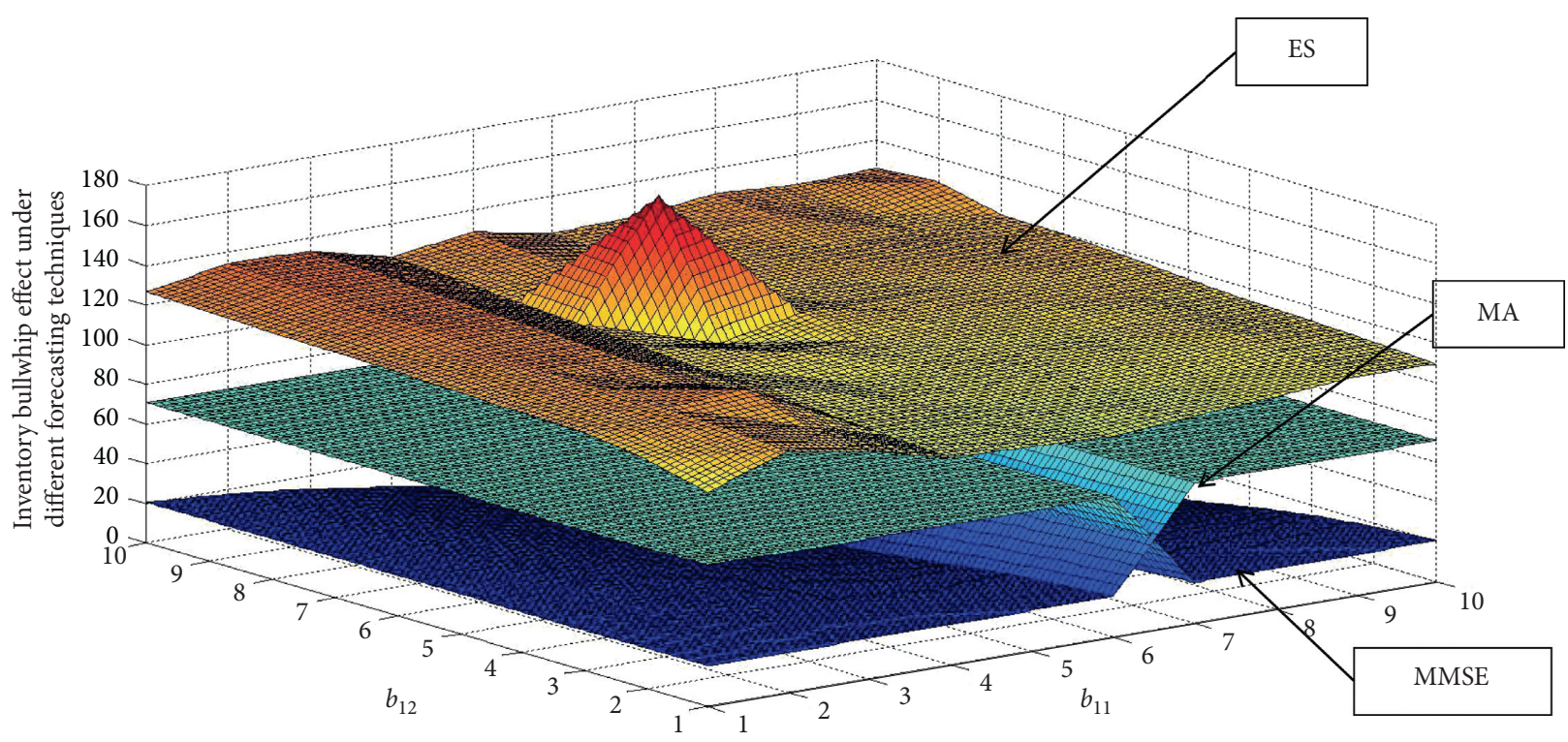

FIGURE 11: The impact of $b_{11}$ and $b_{12}$ on the inventory bullwhip effect using different forecasting techniques.

Proposition 2. When the self-price sensitivity coefficient $b_{11}$, the cross-price sensitivity coefficient $b_{12}$, the price autoregressive coefficients $\rho_{1}$ and $\rho_{2}$, and the market share $\psi$ satisfy the following condition, $R 1$ can control the inventory bullwhip effect using the MMSE technique. Otherwise, R1 should select the MA technique.

$$
\begin{aligned}
& {\left[2\left(\frac{\rho_{1}}{1-\rho_{1}}\right)^{2}+2 \frac{\rho_{1}}{1-\rho_{1}}\right]-2\left(1+\frac{\rho_{1}}{1-\rho_{1}}\right)\left(\frac{\rho_{1}}{1-\rho_{1}}\right)\left\{b_{11} b_{12}\left(\rho_{1}^{k}+\rho_{2}^{k}\right) \frac{\psi(1-\psi)}{1-\rho_{1} \rho_{2}}\right\}} \\
& \quad<2 b_{11} b_{12}\left(\left(\rho_{1} \frac{\rho_{2}\left(1-\rho_{1}\right)}{1-\rho_{1} \rho_{2}} \frac{\rho_{2}\left(1-\rho_{2}^{l_{1}}\right)}{1-\rho_{2}^{2}}\right)+\left(\rho_{2} \frac{\rho_{1}\left(1-\rho_{2}\right)}{1-\rho_{1} \rho_{2}} \frac{\rho_{1}\left(1-\rho_{1}^{l_{1}}\right)}{1-\rho_{1}^{2}}\right)\right) .
\end{aligned}
$$




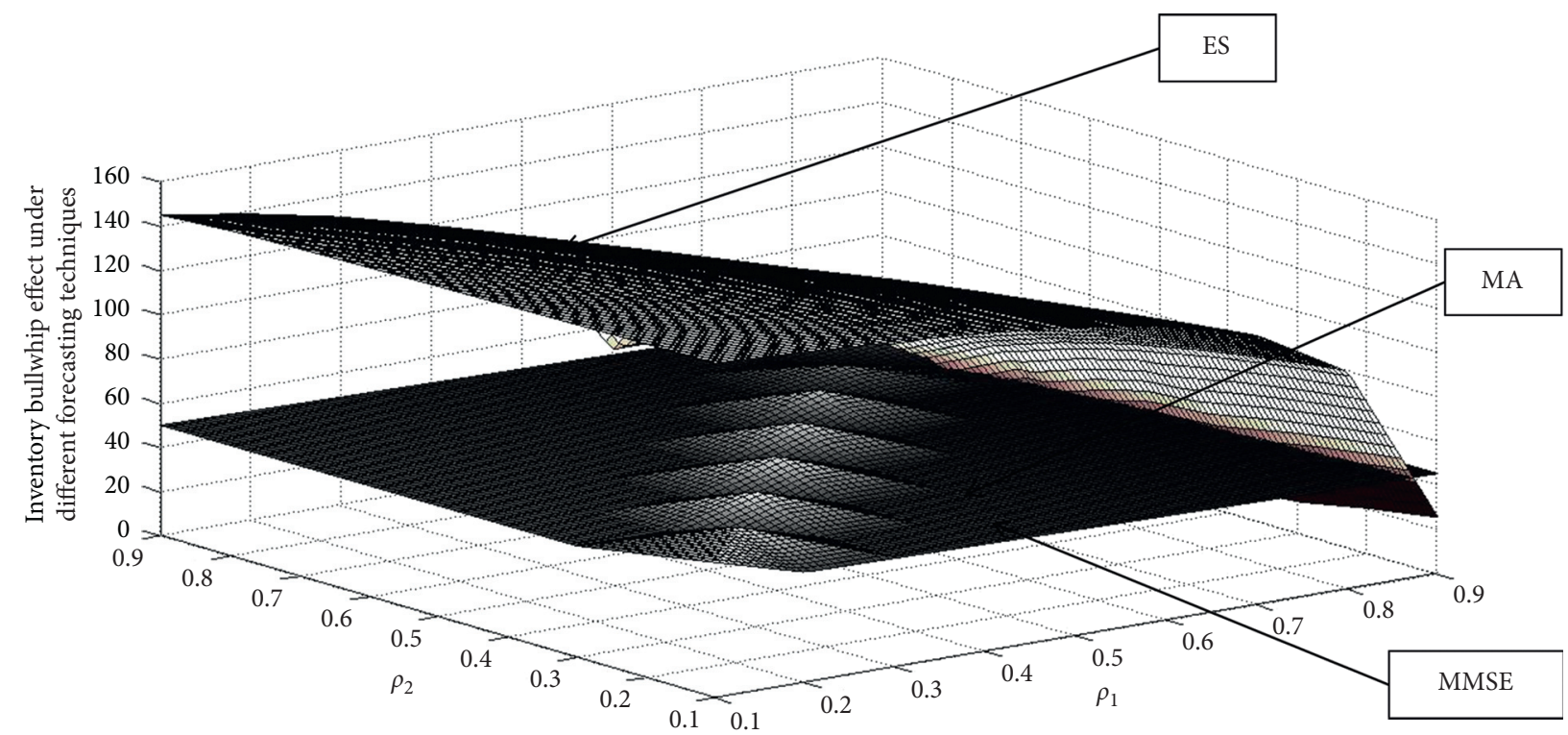

FIGURE 12: The impact of $\rho_{1}$ and $\rho_{2}$ on the inventory bullwhip effect using different forecasting techniques.

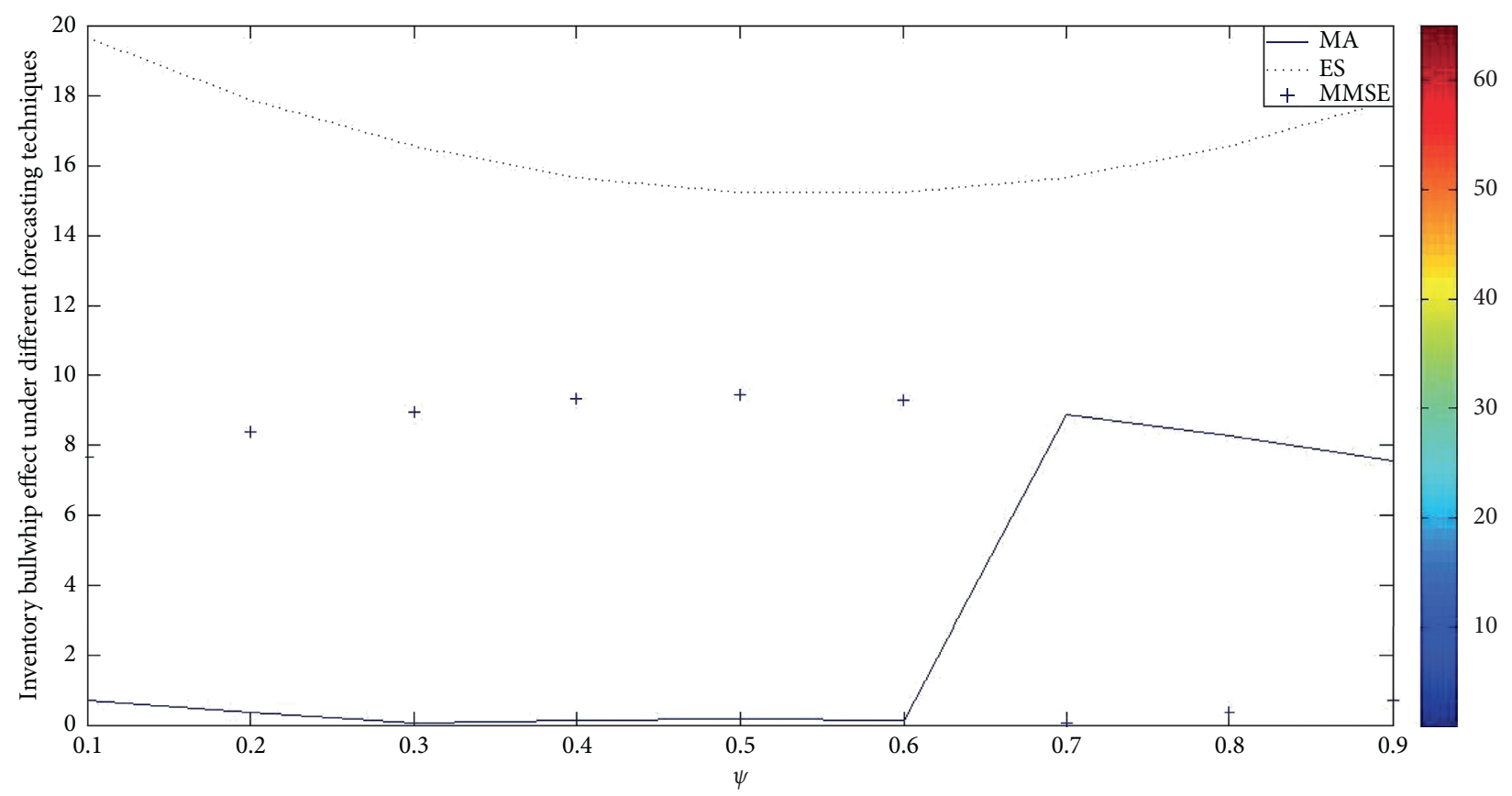

Figure 13: The impact of $\psi$ on the inventory bullwhip effect using different forecasting techniques.

We found that the potential market demand $a_{1}$ has no impact on R1's inventory bullwhip effect. Thus, the potential market demand cannot influence R1's choice of the forecasting technique. On the contrary, when the self-price sensitivity coefficient $b_{11}$, the cross-price sensitivity coefficient $b_{12}$, the price autoregressive coefficients $\rho_{1}$ and $\rho_{2}$, and the market share $\psi$ satisfy Proposition 2, R1 can control the inventory bullwhip effect using the MMSE technique. Otherwise, R1 should select the MA technique.

From the above analysis, we can obtain the following managerial insights:
Managerial Insight 1 . Figure 8 clearly illustrates that there is a positive relationship between $b_{12}$ and BE. In other words, the larger the value of the cross-price sensitivity coefficient, the greater the competition between the two supply chains. As a result, the fluctuation in demand increases. Thus, in terms of the operational and management activities of the firm, the firm should seek to reduce the competitive relationship and maintain a positive cooperative relationship.

Managerial Insight 2. As shown in Figure 10, there is a positive relationship between $\psi$ and BE. The reason for 
this phenomenon is that the larger the probability that customers will choose both retailers' products, the more difficult it is for the two retailers to forecast customer demand. Thus, this will lead to greater uncertainty with respect to the retailer's order.

Managerial Insight 3. As Figure 11 clearly shows, there is a positive relationship between $b_{12}$ and IBE. In other words, the larger the value of the cross-price sensitivity coefficient, the greater the competition between the two supply chains, and the fluctuation in the inventory level increases. Thus, the firms should reduce the competitive relationship and maintain a positive cooperative relationship between different firms.

Managerial Insight 4. As shown in Figure 13, there is a positive relationship between $\psi$ and IBE.

As shown in Managerial Insight 2, the larger the probability that customers will choose both retailers' products, the larger the bullwhip effect of the two retailers. As a result, this will lead to more uncertainty in the retailer's inventory. Thus, the bullwhip effect will increase for both retailers.

Managerial Insight 5. As shown in the simulation result, with the increase in relevant variables (i.e., the self-price sensitivity coefficient and the cross-price sensitivity coefficient), the retailer's bullwhip effect and the inventory bullwhip effect become larger and larger. Moreover, the fluctuation in the inventory level is higher than the fluctuation in the level of demand. This means that the retailer should control the volatility of the demand so that the volatility of the inventory level can be controlled.

\section{Conclusion}

In this paper, we researched the impact of different forecasting techniques and market share on the inventory bullwhip effect in two parallel supply chains which include one manufacturer and one retailer in each supply chain. We assumed that the two retailers order products from two manufacturers using the order-up-to strategy and that the two retailers predict customer demand using different forecasting techniques. First, we developed a quantitative model of the inventory bullwhip effect based on the bullwhip effect and by means of inventory. We then analyzed the impact of the self-price sensitivity coefficient, the cross-price sensitivity coefficient, the different forecasting techniques, and the market share on the inventory bullwhip effect. Finally, we analyzed the condition under which different forecasting techniques and the market share can increase or reduce the inventory bullwhip effect. We reached the following conclusions:

(1) The bullwhip effect can achieve the lowest level under the MMSE technique when the value of the self-price sensitivity coefficient $b_{11}$ is lower than 7 . On the contrary, the bullwhip effect can achieve the lowest level using the MA technique when $b_{11}$ is higher than 7 . The bullwhip effect can reach the lowest level using the MMSE technique when the price autoregressive coefficient $\rho_{1}<0.8$.

(2) The bullwhip effect can achieve the lowest level using the MA technique when the value of the market share $\psi$ is lower than 0.6. Otherwise, the bullwhip effect can achieve the lowest level using the MMSE technique when $\psi$ is higher than 0.6. Moreover, $\Delta \mathrm{BE}_{\text {retailer-1 }}=\mathrm{BE}_{\text {retailer-1 }}^{\mathrm{MMSE}}-\mathrm{BE}_{\text {retailer-1 }}^{\mathrm{MA}}$, when $\sigma_{1}^{2}=\delta_{1}^{2}$ and $\sigma_{2}^{2}=\delta_{2}^{2}$.

(3) The MMSE forecasting technique can reduce the lead-time demand forecast error to the largest extent, and the inventory bullwhip effect can achieve the lowest level using the MMSE technique.

(4) R1 can reduce the inventory bullwhip effect using the MA method when the self-price sensitivity coefficient $b_{11}$ is lower than 5.5 , the price autoregressive coefficient $\rho_{1}$ is lower than 0.45 , and the market share $\psi$ is lower than 0.4 .

Our research suggests that the manager should strengthen cooperation with the partner. If two products are substitutable or two supply chains are competitors, they should reduce the competitive relationship and maintain positive cooperative relationship. Moreover, the firm should forecast the market demand using the mean square error forecasting method under some conditions. On the contrary, the company can do this by using other forecasting methods under some conditions. In this paper, we only researched the inventory bullwhip effect in two parallel supply chains. In the future, we will discuss the inventory bullwhip effect in a supply chain network which includes multiple suppliers, multiple manufacturers, multiple distributors, and multiple retailers. Lastly, we should point out that many suppliers or retailers build the network selling channel to sell products by means of electronic commerce. There is a competitive relationship between the network selling channel and the traditional entity selling channel. In the future, we will discuss the impact of different selling channels on the inventory bullwhip effect.

\section{Data Availability}

The data used to support the findings of this study are available from the corresponding author upon request.

\section{Conflicts of Interest}

The authors declare that they have no conflicts of interest.

\section{Acknowledgments}

This work was supported by the Project of the China Scholarship Council (no. 201706980022), Outstanding Doctor Scientific Research Fund Project in Jiangsu Normal University (nos. 18XWRS008 and 19XFRS002), the Natural Science Foundation of University in Jiangsu Province (no. 19KJB120006), and the Priority Academic Program Development of Jiangsu Higher Education Institutions (PADP). 


\section{References}

[1] H. L. Lee, V. Padmanabhan, and S. Whang, "Information distortion in a supply chain: the bullwhip effect," Management Science, vol. 43, no. 4, pp. 546-558, 1997.

[2] H. L. Lee, V. Padmanabhan, and S. Whang, "The bullwhip effect in supply chains," Sloan Management Review, vol. 38, no. 3, pp. 93-102, 1997.

[3] F. Chen, Z. Drezner, J. K. Ryan, and D. Simchi-Levi, "Quantifying the bullwhip effect in a simple supply chain: the impact of forecasting, lead times, and information," Management Science, vol. 46, no. 3, pp. 436-443, 2000.

[4] F. Chen, J. K. Ryan, D. Simchi-Levi, and D. Smith-Levi, "The impact of exponential smoothing forecasts on the bullwhip effect," Naval Research Logistics, vol. 47, no. 4, pp. 269-286, 2000.

[5] H. T. Luong, "Measure of bullwhip effect in supply chains with autoregressive demand process," European Journal of Operational Research, vol. 180, no. 3, pp. 1086-1097, 2007.

[6] H. T. Luong and N. H. Phien, "Measure of bullwhip effect in supply chains: the case of high order autoregressive demand process," European Journal of Operational Research, vol. 183, no. 1, pp. 197-209, 2007.

[7] Y. Ma, N. Wang, A. Che, Y. Huang, and J. Xu, "The bullwhip effect on product orders and inventory: a perspective of demand forecasting techniques," International Journal of Production Research, vol. 51, no. 1, pp. 281-302, 2013.

[8] H. L. Lee, K. C. So, and C. S. Tang, "The value of information sharing in a two-level supply chain," Management Science, vol. 46, no. 5, pp. 626-643, 2000.

[9] L. C. Alwan, J. J. Liu, and D. Q. Yao, "Stochastic characterization of upstream demand processes in a supply chain," IEEE Transactions, vol. 35, no. 9, pp. 207-219, 2003.

[10] W. Hausman and J. Miyaoka, "How a base stock policy using stale forecasts provides supply chain benefits," Manufacturingeservice Operations Management, vol. 51, no. 10, pp. 3085-3116, 2004.

[11] H. Liu and P. Wang, "The simulation of the bullwhip effect in multi-level supply chain based on the ARMA $(1,1)$ demand model," Journal of System Simulation, no. 6, pp. 3253-3257, 2008.

[12] G. Gaalman and S. M. Disney, "State space investigation of bullwhip with ARMA $(1,1)$ demand processes," International Journal of Production Economics, vol. 104, no. 2, pp. 327-339, 2007.

[13] G. Gaalman and S. M. Disney, "On bullwhip in a family of order-up-to policies with $\operatorname{ARMA}(2,2)$ demand and arbitrary lead-times," International Journal of Production Economics, vol. 121 , no. 2 , pp. 454-463, 2009.

[14] C. C. Holt, F. Modigliani, J. Muth, and H. Simon, Planning Production, Inventories and the Work Force, Prentice-Hall, Upper Saddle River, NJ, USA, 1960.

[15] A. S. Blinder, "Retail inventory investment and business fluctuations," Booking Paper on Economics Activity, vol. 2, no. 43, pp. 443-505, 1981.

[16] M. Baganha and M. Cohen, "The stabilizing effect on inventory in supply chains," Operational Research, vol. 46, no. 13 , pp. 572-583, 1998.

[17] D. R. Towill, "Dynamic analysis of an inventory and order based production control system," International Journal of Production Research, vol. 15, no. 3, pp. 369-383, 2000.

[18] X. Y. Huang and Z. Lu, "Distribution-centre supply chain model and bullwhip effect $H_{\propto}$ control," Journal of Chinese Management Science, vol. 6, no. 3, pp. 86-93, 2003.
[19] S. M. Disney, D. R. Towill, and W. Van De Velde, "Variance amplification and the golden ratio in production and inventory control," International Journal of Production Economics, vol. 90, no. 3, pp. 295-309, 2004.

[20] R. G. Ingalls, B. L. Foote, and A. Krishnamoorthy, "Reducing the bullwhip effect in supply chains with control-based forecasting," International Journal of Simulation and Process Modelling, vol. 1, no. 1/2, pp. 90-110, 2005.

[21] Y. Feng and J. H. Ma, "The research on the bullwhip effect based on," O-S Control Theory, vol. 11, no. 4, pp. 50-56, 2008.

[22] F. Costantino, G. DiGravio, A. Shaban, and M. Tronci, "SPC forecasting system to mitigate the bullwhip effect and inventory variance in supply chains," Expert System with Application, vol. 42, no. 5, pp. 1773-1787, 2015.

[23] M. Udenio, J. C. Fransoo, and R. Peels, "Destocking, the bullwhip effect, and the credit crisis: empirical modeling of supply chain dynamics," International Journal of Production Economics, vol. 160, no. 2, pp. 34-46, 2015.

[24] H.-K. Kim and J. K. Ryan, "The cost impact of using simple forecasting techniques in a supply chain," Naval Research Logistics, vol. 50, no. 5, pp. 388-411, 2003.

[25] Z. Michna, S. M. Disney, and N. Peter, "The impact of stochastic lead times on the bullwhip effect under correlated demand and moving average forecasts," Omega, vol. 93, no. 2020, pp. 1-11, 2020.

[26] P. Erica, A. Arianna, Z. Giulio, and E. B. John, "The impact of demand parameter uncertainty on the bullwhip effect," European Journal of Operational Research, vol. 283, no. 2020, pp. 94-107, 2020.

[27] W. Holland and M. S. Sodhi, "Quantifying the effect of batch size and order errors on the bullwhip effect using simulation," International Journal of Logistics Research and Applications, vol. 7, no. 3, pp. 251-261, 2004.

[28] X. Zhang, "The impact of forecasting methods on the bullwhip effect," International Journal of Production Economics, vol. 88, no. 1, pp. 15-27, 2004.

[29] X. Zhang, "Delayed demand information and dampened bullwhip effect," Operations Research Letters, vol. 33, no. 3, pp. 289-294, 2005.

[30] T. Hosoda and S. M. Disney, "On variance amplification in a three-echelon supply chain with minimum mean square error forecasting," Omega, vol. 34, no. 4, pp. 344-358, 2006.

[31] Y. J. Lu, X. W. Tang, and Y. Zhang, "The fractal characteristics of the bullwhip effect in supply chain," Journal of System Engineering, vol. 21, no. 5, pp. 463-469, 2006.

[32] G. P. Cachon, T. Randall, and G. M. Schmidt, "In search of the bullwhip effect," Manufacturing \& Service Operations Management, vol. 9, no. 4, pp. 457-479, 2007.

[33] H. Liu and P. Wang, "ARMA $(1,1)$ based on the needs of multilevel supply chain bullwhip effect simulation," Journal of System Simulation, vol. 21, no. 6, pp. 3253-3257, 2008.

[34] I. Kim and M. Springer, "Measuring endogenous supply chain volatility: beyond the bullwhip effect," European Journal of Operational Research, vol. 189, no. 1, pp. 172-193, 2008.

[35] M. Springer and I. Kim, "Managing the order pipeline to reduce supply chain volatility," European Journal of Operational Research, vol. 203, no. 2, pp. 380-392, 2010.

[36] M. Coppini, T. Rossi, and F. Strozzi, "Bullwhip effect and inventory oscillations analysis using the beer game model," International Journal of Production Research, vol. 48, no. 13, pp. 3943-3956, 2010.

[37] Y. Rossignoli, N. Wang, A. Che, Y. Huang, and J. Xu, "The bullwhip effect under different information-sharing settings: a perspective on price-sensitive demand that incorporates price 
dynamics," International Journal of Production Research, vol. 51, no. 10, pp. 3085-3116, 2013.

[38] F. Costantino, G. Di Gravio, A. Shaban, and M. Tronci, “The impact of information sharing and inventory control coordination on supply chain performances," Computers \& Industrial Engineering, vol. 76, no. 4, pp. 292-306, 2014.

[39] L. Robert and M. Haim, "Production smoothing and the bullwhip effect," Manufacturing and Service Operations, vol. 17, no. 2, pp. 208-220, 2015.

[40] K. Hossein, H. S. Moattar, and M. R. Marjani, “The bullwhip effect in a 3-stage supply chain considering multiple retailers using a moving average method for demand forecasting," Applied Mathematical Modelling, vol. 40, no. 1, pp. 89348951, 2016.

[41] K. Marieh and A. S. Mohsen, "Analyzing the behavior of the bullwhip effect considering different distribution systems," Applied Mathematical Modelling, vol. 59, no. 1, pp. 319-340, 2018.

[42] T. Y. Zhu, J. Balakrishnan, and J. C. Giovani, "Bullwhip effect in the oil and gas supply chain: a multiple-case study," International Journal of Production Economics, vol. 224, no. 2020, pp. 1-14, 2020.

[43] X. L. Zhang and Y. Zhao, "The impact of external demand information on parallel supply chains with interacting demand," Production and Operations Management, vol. 19, no. 4, pp. 463-479, 2010.

[44] X.-q. Zhang and X.-g. Yuan, "The system dynamics model in electronic products closed-loop supply chain distribution network with three-way recovery and the old-for-new policy," Discrete Dynamics in Nature and Society, vol. 2016, Article ID 4074710, 10 pages, 2016.

[45] X. G. Yuan and N. Zhu, "Bullwhip effect analysis in two-level supply chain distribution network using different demand forecasting method," Asia Pacific Journal of Operational Research, vol. 33, no. 3, pp. 46-69, 2016.

[46] S. Zhang and M. Zhang, "Mitigation of bullwhip effect in closed-loop supply chain based on fuzzy robust control approach," Complexity, vol. 2020, Article ID 1085870, 17 pages, 2020.

[47] J. Ma and B. Bao, "Research on bullwhip effect in energyefficient air conditioning supply chain," Journal of Cleaner Production, vol. 143, no. 1, pp. 854-865, 2017.

[48] H. J. Vassian, "Application of discrete variable servo theory to inventory control," Journal of the Operations Research Society of America, vol. 3, no. 3, pp. 272-282, 1955.

[49] G. E. P. Box and G. M. Jenkins, Time Series Analysis: Forecasting and Control, Prentice Hall, Englewood Cliffs, NJ, USA, 3rd edition, 1994. 